\title{
1 Hierarchical Bayesian Modeling of the Relationship between Task 2 Related Hemodynamic Responses and Neuronal Excitability: a 3 Simultaneous fNIRS/TMS Study
}

5 Zhengchen $\mathrm{Cai}^{1,2}$, Giovanni Pellegrino ${ }^{3,4}$, Jean-Marc Lina ${ }^{5,6}$, Habib Benali ${ }^{2}$, Christophe

6 Grova $^{1,2,4,6}$

$8{ }^{1}$ Department of Physics, Concordia University, Montréal, Québec, Canada

9 PERFORM Centre, Concordia University, Montréal, Québec, Canada

$10{ }^{2}$ PERFORM Centre, Concordia University, Montréal, Québec, Canada

$11{ }^{3}$ Neurology and Neurosurgery Department, Montreal Neurological Institute, McGill

12 University, Montréal, Québec, Canada

$13{ }^{4}$ Multimodal Functional Imaging Lab, Biomedical Engineering Department, McGill

14 University, Montréal, Québec, Canada

$15{ }^{5}$ Département de Génie Electrique, École de Technologie Supérieure, Montréal, Québec,

16 Canada

$17{ }^{6}$ Centre De Recherches En Mathématiques, Montréal, Québec, Canada

\section{Correspondence:}

Zhengchen Cai,

Multimodal Functional Imaging Lab,

Department of Physics and PERFORM Centre, Concordia University. Loyola Campus, Office SP 365.23

7141 Sherbrooke Street West, Montreal, QC, H4B 1R6, Canada

Phone: (514) 848-2424 ext.3393

Corresponding author emails: zhengchen.cai@mail.concordia.ca; zhengchen.cai@gmail.com 


\section{Abstract:}

39 Background: Investigating the relationship between task-related cortical hemodynamic

40 activity and brain excitability is challenging because it requires simultaneous measurement of

41 brain hemodynamic activity while applying non-invasive brain stimulation. There is also

42 considerable inter-/intra-subject variability which both brain excitability and task-related

43 hemodynamic responses are associated with. Here we proposed hierarchical Bayesian

44 modeling to taking into account variability in the data at the individual and group levels,

45 aiming to provide accurate and reliable statistical inferences on this research question.

46 Methods: We performed a study on 16 healthy subjects with simultaneous Paired

47 Associative Stimulation (Inhibitory PAS10, Excitatory PAS25, Sham) and functional Near-

48 Infrared Spectroscopy (fNIRS) targeting the primary motor cortex (M1). PAS was applied to

49 modulate the cortical function and induce plasticity. Before and after each intervention

50 cortical excitability was measured by motor evoked potentials (MEPs), and the motor task-

51 related hemodynamic response was measured using fNIRS. We constructed three models to

52 encode 1) PAS effects on the M1 excitability; 2) PAS effects on the whole-time course of

53 fNIRS hemodynamic responses to finger tapping tasks, and 3) the correlation between PAS

54 effects on M1 excitability and PAS effects on task-related hemodynamic responses.

55 Results: Significant increase of the cortical excitability was found after PAS25, whereas a

56 small reduction of the cortical excitability was shown after PAS10 and no changes after

57 sham. We found PAS effects on finger tapping evoked $\mathrm{HbO} / \mathrm{HbR}$ within $\mathrm{M} 1$, around the peak

58 of the hemodynamic time courses. Both $\mathrm{HbO}$ and $\mathrm{HbR}$ absolute amplitudes increased after

59 PAS25 and decreased after PAS10. Cortical excitability changes and task-related $\mathrm{HbO} / \mathrm{HbR}$

60 changes showed a high probability of being positively correlated, 0.77 and 0.79 , respectively.

61 The corresponding Pearson's correlations were 0.58 ( $\mathrm{p}<.0001, \mathrm{HbO}$ with MEP) and 0.56

62 ( $\mathrm{p}<.001, \mathrm{HbR}$ with MEP), respectively.

63 Conclusion: Benefiting from this original Bayesian data analysis, our results showed that

64 PAS modulates task-related cortical hemodynamic responses in addition to M1 excitability.

65 The fact that PAS effects on hemodynamic response were exhibited mainly around the peak

66 of the hemodynamic time course may indicate that the intervention only increases metabolic

67 demanding rather than modulating hemodynamic response function per se. Moreover, the

68 positive correlation between PAS modulations of excitability and hemodynamic brings

69 insights to understand the fundamental properties of cortical function and cortical excitability. 
71 Keywords: Paired Associative Stimulation (PAS), Excitability, Maximum Entropy on the

72 Mean (MEM), Finger Tapping, Near-Infrared Spectroscopy (NIRS), Transcranial Magnetic

73 Stimulation (TMS), Bayesian Data Analysis.

74

75 Highlights:

76 - Hierarchical Bayesian Modeling of PAS effects on M1 excitability and hemodynamic

77 - Demonstrated PAS effects on the whole time-course of task-related hemodynamic

78 - Increased/reduced task-related hemodynamic corresponds to higher/lower M1

79 excitability

80 - High probability of positive relationships between excitability and $\mathrm{HbO} / \mathrm{HbR}$ changes 


\section{Introduction}

83 The association between hemodynamic response to a task and excitability of the

84 corresponding cortical region helps to understand the relationship between cortical metabolic

85 demand and cortical readiness. This knowledge might be useful to expand the field of

86 application of non-invasive brain stimulation for treating brain disorders in which modulation

87 of hemodynamic activity is desired. Currently, there are several non-invasive brain

88 stimulation techniques capable of interacting with cortical function and are potentially useful

89 to treat neurological conditions. One of the eldest and better-known techniques is repetitive

90 Transcranial Magnetic Stimulation (rTMS): TMS pulses repeated in a train following a

91 certain frequency induce Long-Term Potentiation (LTP)-like and Long-Term Depression

92 (LTD)-like plasticity which are typically measured as changes of cortical excitability

93 (Ridding and Rothwell, 2007; Di Pino et al., 2014). Another way to induce plasticity relies on

94 the concept of Spike Timing Dependent Plasticity (STDP, Levy and Steward, 1983; Rossini

95 et al., 2015) and is entitled Paired Associative Stimulation (PAS) (Mariorenzi et al., 1991;

96 Stefan, 2000). PAS consists of pairs of cortical TMS and peripheral electrical Median Nerve

97 Stimulation (MNS) delivered with proper timing: around $25 \mathrm{~ms}$ or $10 \mathrm{~ms}$ Interstimulus

98 Intervals (ISI) to excite (PAS25) or to inhibit (PAS10) primary motor cortical areas,

99 respectively. The peak-to-peak amplitude of Motor Evoked Potentials (MEPs) measured by

100 electromyography (EMG) on the hand muscle is often used to assess the cortical excitability

101 while delivering single pulse TMS (spTMS) on top of the primary motor cortex (Suppa et al.,

102 2017).

103 In animal studies, by applying invasive optical imaging, Allen et al. (2007) demonstrated that

104 low-frequency repetitive rTMS, known for reducing cortical excitability, on cat's visual

105 cortex induces an immediate increase of tissue oxygenation followed by a prolonged

106 reduction of oxygenation lasting approximately 2 minutes. More recently, a study on healthy

107 rats combining functional Magnetic Resonance Imaging (fMRI) and proton Magnetic

108 Resonance Spectroscopy (MRS) showed increases in resting-state connectivity (e.g.,

109 Interoceptive/default model network, cortico-striatal-thalamic network and Basal ganglial

110 network), GABA, glutamine and glutamate levels following high-frequency rTMS (known

111 for increasing cortical excitability) and reduced connectivity and glutamine levels after low-

112 frequency rTMS stimulations (Seewoo et al., 2019).

113 In human studies, similar investigations become more challenging which require the 114 combination of non-invasive neuroimaging and non-invasive brain stimulation approaches. 
115 For instance, fMRI (Bandettini et al., 1992; Kwong et al., 1992; Glover, 2011) is a widely

116 used modality to measure the hemodynamic activity and can measure the hemodynamic

117 fluctuations related to TMS interventions (Navarro De Lara et al., 2015; Tik et al., 2017).

118 However, simultaneous fMRI/TMS acquisitions require specific MRI coil and fMRI

119 sequences (Navarro De Lara et al., 2015; Wang et al., 2017). This is the reason why most

120 studies consisted in fMRI sessions before and after TMS interventions, following a so-called

121 offline approach (Siebner et al., 2009).

122 Alternatively, functional Near-InfraRed Spectroscopy (fNIRS) non-invasively measures

123 fluctuations of both oxygenated- and deoxygenated-hemoglobin (i.e., $\mathrm{HbO}$ and $\mathrm{HbR}$ )

124 concentration changes in the human brain with a high temporal resolution (Jöbsis, 1977;

125 Scholkmann et al., 2014). fNIRS relies on optical absorption which is insensitive to

126 electromagnetic fields, as opposed to fMRI. Therefore, fNIRS appears as an interesting

127 alternative offering better compatibility for simultaneous acquisition during TMS (Curtin et

128 al., 2019). In Cai et al., 2021b, we conducted a simultaneous PAS-fNIRS study to investigate

129 the relationship between motor task-evoked cortical hemodynamic response and M1

130 excitability. We opted for PAS rather than rTMS for multiple reasons. A previous

131 simultaneous TMS/fNIRS study reported that physiological fluctuations of respiration and

132 heart rate are largely influenced by trains of TMS pulses (Näsi et al., 2011). In other words,

133 TMS trains may influence both systemic hemodynamic (scalp signal) and cortical

134 hemodynamic responses. Second, the frequency of stimulation pairs in PAS is $0.1 \mathrm{~Hz}$ or less

135 (Suppa et al., 2017), therefore PAS intervention is likely introducing significantly less or

136 even no systemic physiological fluctuations when compared to rTMS. Third, when several

137 stimulations with multiple effects are applied (excitatory, inhibitory, sham), PAS offers

138 stimulations that are similar with respect to duration and frequency across conditions,

139 allowing a more balanced and cleaned experiment design.

140 Beyond the technical difficulties of addressing the relationship between excitability and

141 hemodynamic activity, one main challenge lies in the high intra- and inter-subject variability

142 of both the effects of brain stimulation and the hemodynamic activity associated with a task.

143 The ability of PAS in eliciting significant changes in cortical excitability has been replicated

144 by several studies (Stefan, 2000; Wolters et al., 2005; Tsang et al., 2015; Lee et al., 2017;

145 Suppa et al., 2017), but some studies have observed that only 39\% of subjects showed

146 expected MEP amplitude increase after conducting PAS25 (López-Alonso et al., 2014) and

147 similar PAS efficiency (lower than 50\%) has been suggested in a review study (Suppa et al., 
148 2017). Similarly, inter-subject variability of the task-evoked hemodynamic response has also

149 been reported, whether measured using fMRI (Witt et al., 2008) or fNIRS (Novi et al., 2020).

150 These variability issues may explain the negative findings on the correlation between cortical

151 excitability and hemodynamic activities. For instance, Kriváneková et al., (2013) investigated

152 the relationship between the primary motor cortex (M1) excitability and Blood-Oxygen-

153 Level-Dependent (BOLD) signal using PAS stimulation and "offline" fMRI acquisitions.

154 They reported no significant correlation between PAS effects on task-related BOLD response

155 and its effects on M1 excitability. In our previous study (Cai et al., 2021b), using fNIRS and

156 PAS we first found a significant positive correlation between fluctuations of cortical

157 excitability (represented by MEP) and the fluctuations of $\mathrm{HbO}$ activity. However, when

158 further investigating the relationship between PAS effects on task-related $\mathrm{HbO} / \mathrm{HbR}$ changes

159 (estimated using the $\mathrm{HbO}$ ratio, $\mathrm{HbR}$ respectively, calculated as the post- over pre-

160 intervention amplitudes) and its effects on M1 excitability (estimated using the ratio of MEP),

161 we also found no significant correlation. Therefore, it seems essential to carefully take into

162 account intrinsic variability of both cortical excitabilities elicited by non-invasive brain

163 stimulation and hemodynamic responses to tasks, when investigating the correlation between

164 both effects.

165 To conduct accurate and robust investigations of PAS elicited cortical excitability (measured 166 using MEP) and hemodynamic responses to finger tapping task (measured using $\mathrm{HbO} / \mathrm{HbR}$ ), 167 we propose to study the variability of data within a hierarchical Bayesian model to infer PAS 168 effects on both cortical excitability and hemodynamic response, as well as their correlation.

169 Hierarchical Bayesian modeling allows taking into account heterogeneity of the variables of 170 interest (MEP, $\mathrm{HbO} / \mathrm{HbR}$ ) at every stage (inter-/intra-subject, intervention type) of the 171 analysis (Papaspiliopoulos et al., 2007; Betancourt and Girolami, 2015). Moreover, when 172 considering a hierarchical structure, partial pooling can reduce the uncertainty of estimated 173 parameters (Gelman et al., 2013; McElreath, 2020). This means that the group-level and 174 individual-level estimations could inform each other to regularize the uncertainty of each 175 parameter. In this context, Bayesian data analysis allows estimating the statistical expectation 176 of each parameter of the model by sampling the joint posterior distributions. Thanks to the 177 developments in Bayesian data analysis workflow during the last decade, Bayesian inferences 178 have become more accessible and can provide accurate and reliable estimations of the 179 posterior distribution. For instance, the most recent implementations of the Hamiltonian 180 Monte Carlo (HMC) algorithm (Duane et al., 1987) called the dynamic HMC (Betancourt, 
181 2017, 2019) is available as an open-source Bayesian statistical modeling and computation

182 platform called Stan (Stan Development Team, 2020a). This technique not only accurately

183 and efficiently samples the joint posterior distribution, but also provides robust estimations

184 by quantitatively diagnosing pathological behaviors of Markov Chain Monte Carlo (MCMC)

185 chains that are used to sample the joint posterior distributions (Betancourt and Girolami,

186 2015; Betancourt, 2017).

187 Considering the above advantages of the Bayesian approach, in this study, we applied a 188 Bayesian data analysis workflow (Gabry et al., 2019; Gelman et al., 2020b) on our 189 TMS/fNIRS dataset rather than the conventional analysis conducted in Cai et al., 2021b, to 190 further investigate the relationship between the PAS effects task-related hemodynamic 191 responses and its effects on M1 excitability. We hypothesize that enhanced brain excitability 192 should be associated with higher hemodynamic activity elicited by a finger tapping task, and 193 decreased excitability should be associated with a reduced hemodynamic response to the task.

194 We first summarized the study design and data acquisition. Data preprocessing was then 195 conducted to prepare the inputs of our Bayesian framework. We evaluated the relevance of 196 three hierarchical models to investigate: 1) PAS effects on M1 excitability measured using 197 MEP; 2) PAS effects on the whole-time course of task-related hemodynamic responses 198 measured using fNIRS, and 3) the correlation between PAS modulated excitability changes 199 and PAS modulated hemodynamic changes. The variability of each measurement was 200 carefully considered in each model and at each level (i.e., at the individual and group levels) 201 to conduct reliable estimations of the intervention effects and correlations. Statistical 202 inferences were made via posterior predictive simulations (McElreath, 2020). Diagnostic of 203 the models were conducted to ensure the robustness of the estimated posterior distributions.

\section{Material and methods}

\section{$205 \quad 2.1$ Study design and subjects}

206 Nineteen subjects (19 - 35 years old, male and right-handed) with no history of neurological 207 disorders and no medications acting on the central nervous system were selected to 208 participate in the study. We only included male participants in order to minimize the 209 confounding of cortical excitability changes due to the menstrual cycle (Hattemer et al., 210 2007; Lee et al., 2017). This study was approved by the Central Committee of Research 211 Ethics of the Minister of Health and Social Services Research Ethics Board (CCER), Québec, 212 Canada. All subjects signed written informed consent prior to participation. They also 
213 underwent a screening procedure to confirm no contraindications to MRI or TMS (Rossi et

214 al., 2009; Suppa et al., 2017). Subjects were instructed to have a regular sleep cycle for the

215 days and not to take caffeine for at least 90 minutes before the data acquisition.

216 The experiment paradigm of this study is illustrated in Fig.1.a. To modulate M1 cortical 217 excitability and for inducing brain plasticity, three different intervention sessions were 218 performed at least two days apart to minimize carryover effects. Each session consisted of 219 five time-ordered sections, defined as follows:

220 1) A block designed finger-tapping task composed of 20 blocks, 10s of finger-tapping followed by 30s 60s of resting was conducted within each block. Subjects were informed to tap their left thumb to the other 4 digits sequentially around 2Hz (Fig.1.a1). This long-range jitter was designed to prevent the task responses from phase locking to the undergoing physiological hemodynamic oscillations (Aarabi et al., 2017), therefore, reducing the physiological confounding on the task-related response at the stage of experiment paradigm design. Tapping onsets/offsets were instructed by auditory cues.

2) An event-related designed single pulse TMS (spTMS) composed of 75 events, jittered from $5 s$ to $25 s$ (Fig.1.a2). TMS procedures were performed with neuronavigation (Brainsight neuro-navigation system - Rogue-Research Inc, Canada) and based on subject specific anatomical MRI. TMS was delivered with a figure-8 coil (Magstim double $70 \mathrm{~mm}$ remote control coil) connected to a Magstim $200^{2}$ stimulator (Magstim Company, U.K.). In order to target M1, the coil was placed tangentially to the scalp and with a $45^{\circ}$ angle to the midline of the head (Fig.1.c), so to maximize stimulation efficiency (Thomson et al., 2013). The individual 'hot spot' was defined for each session as the location with the largest Motor Evoked Potentials (MEPs) amplitude measured on the left thumb (Abductor Pollicis Brevis, APB) using electromyography (EMG). Stimulation intensity was set to $120 \%$ of the resting motor threshold (RMT), defined according to the maximum-likelihood parameter estimation by sequential testing approach (Awiszus et al., 1999; Ah Sen et al., 2017) using MTAT 2.0 (http://www.clinicalresearcher.org/software.html). All TMS procedures followed the recommendations of the International Federation of Clinical Neurophysiology (Rossi et al., 2009) and no participants reported any considerable discomfort or side effects.

3) A PAS session attempted to modulate the M1 cortical excitability (Fig.1.a3). PAS intervention consisted either in PAS25, PAS10 or sham-PAS. PAS was conducted with 
100 pairs of electrical median nerve stimulation (MNS) on the left wrist, followed by TMS pulse delivered over the right M1, with a fixed interval of 10 s between paired stimulations, for a total intervention of 18 minutes, as suggested in Suppa et al., (2017). MNS was delivered with a Digitimer (Digitimer DS7A, U.K) at the left median nerve and with the intensity equals $300 \%$ of the subject-specific perceptual threshold. TMS intensity was the same as the spTMS - $120 \%$ of RMT. After estimating subject-specific N20 response to electrical MNS using bipolar electroencephalogram (EEG) (BrainAmp ExG, Brain Products GmbH, Germany) on CP3 and CP4 electrodes, the interstimulus intervals (ISI) between MNS and TMS were determined to be pre-measured individual N20+5ms for PAS25 and N20-5ms for PAS10 (Carson and Kennedy, 2013). Sham parameters (e.g., MNS intensity, coil position, ISI) were the same as PAS25, but TMS was not delivered, and instead, its sound ('TMS click') was played via a stereo speaker.

4) Repetition of the event-related designed spTMS (Fig.1.a4) after the intervention. By comparing the MEPs measured during pre-intervention and post-intervention sessions, PAS intervention effects on M1 cortical excitability could be assessed.

5) Repetition of the block designed finger tapping task (Fig.1.a5) after the intervention. Similarly, the corresponding effects on task-evoked hemodynamic responses could be estimated by comparing $\mathrm{HbO} / \mathrm{HbR}$ concentration changes measured during preintervention and post-intervention sessions.

\subsection{Data acquisitions}

\subsubsection{Anatomical MRI}

Individual anatomical MRI was acquired to guide TMS and to calculate the head model required for fNIRS acquisition planning and fNIRS reconstructions. A General Electric Discovery MR750 3T scanner at the PERFORM Center of Concordia University, Montréal, Canada, was used to scan: 1) T1-weighted images using the 3D BRAVO sequence $(1 \times 1 \times 1$ $\mathrm{mm}^{3}, 192$ axial slices, $256 \times 256$ matrix) and 2) T2-weighted images using the 3D Cube T2 sequence $\left(1 \times 1 \times 1 \mathrm{~mm}^{3}\right.$ voxels, 168 sagittal slices, $256 \times 256$ matrix $)$.

\subsubsection{Motor Evoked Potentials}

MEPs induced by spTMS pulses were measured to assess the M1 cortical excitability. A BrainAmp ExG bipolar system (BrainAmp ExG, Brain Products GmbH, Germany) was used to record EMG of the right abductor pollicis brevis (APB) muscle, with 2 TECA disposable 
276 20mm disk electromyography (EMG) electrodes attached with a standard belly-tendon

277 montage (Fig1.c).

\section{$278 \quad$ 2.2.3 Functional Near-Infrared Spectroscopy}

279 fNIRS data were acquired to estimate the finger-tapping evoked hemodynamic responses 280 (i.e., $\mathrm{HbO} / \mathrm{HbR}$ ). fNIRS data were acquired at $10 \mathrm{~Hz}$ using a Brainsight fNIRS system 281 (Rogue-Research Inc, Canada), which consists of two wavelengths - 685nm and 830nm. 282 fNIRS optodes were placed to the subject's scalp using a clinical adhesive called collodion 283 (Fig.1.c) to reduce motion artifacts (Yücel et al., 2014; Pellegrino et al., 2016; Machado et 284 al., 2018) and to ensure a better contact with the skin when compared to standard fNIRS caps. 285 A personalized optimal montage developed by our group (Machado et al., 2014, 2018; 286 Pellegrino et al., 2016; Cai et al., 2021c) was used to maximize the sensitivity of fNIRS 287 channels to a predefined region of interest (ROI) - the individual 'hand knob' region (see 288 Fig.1.b) manually defined along the right M1 cortical surface which controls the left hand 289 movement (Raffin et al., 2015). The resulted personalized optimal montage consisted of 3 290 sources and 15 detectors (see Fig.1.b). The distance between each source-detector pair was 291 constrained to range from $2.0 \mathrm{~cm}$ to $4.5 \mathrm{~cm}$. Each source was positioned to construct at least

29213 channels among the 15 detectors ensuring a high spatial overlap between channels, to 293 allow accurate local reconstruction along the cortical surface. A proximity detector was 294 added at the center of 3 sources to record the physiological hemodynamics fluctuations 295 within the scalp. Brainsight neuro-navigation system coregistered with subject specific T1 296 MRI was used to guide the installation and to digitize the position of fNIRS sources and 297 detectors glued at their optimal positions. Additional 150 points were digitized on the head 298 surface to allow accurate montage registration with the anatomical MRI, as a prerequisite for 299 computing the fNIRS forward model. fNIRS data were acquired continuously during the 300 whole experimental session, as described in Fig.1.a.

301 From the nineteen subjects selected for this study, one was excluded due to low sensitivity to 302 TMS and two were excluded because they exhibited poor fNIRS signal qualities. Four 303 subjects dropped out after the first session due to personal reasons, resulting in 16 PAS25, 12 304 PAS10 and 12 sham sessions. Please note that starting from here, we will denote 1) 305 "Session" as one specific acquisition, consisted in any PAS intervention type, of one subject 306 including experiments 1 to 5 as illustrated in Fig.1.a (e.g., PAS25 for Sub01); “Run” as one 307 specific experiment (spTMS or finger tapping), before or after any PAS intervention type, of 308 one subject (e.g., pre-PAS25 spTMS for Sub01); and "Time" to differentiate whether one 
309 specific experiment was conducted before or after the intervention (e.g., pre-PAS25 vs. post-

310 PAS25).
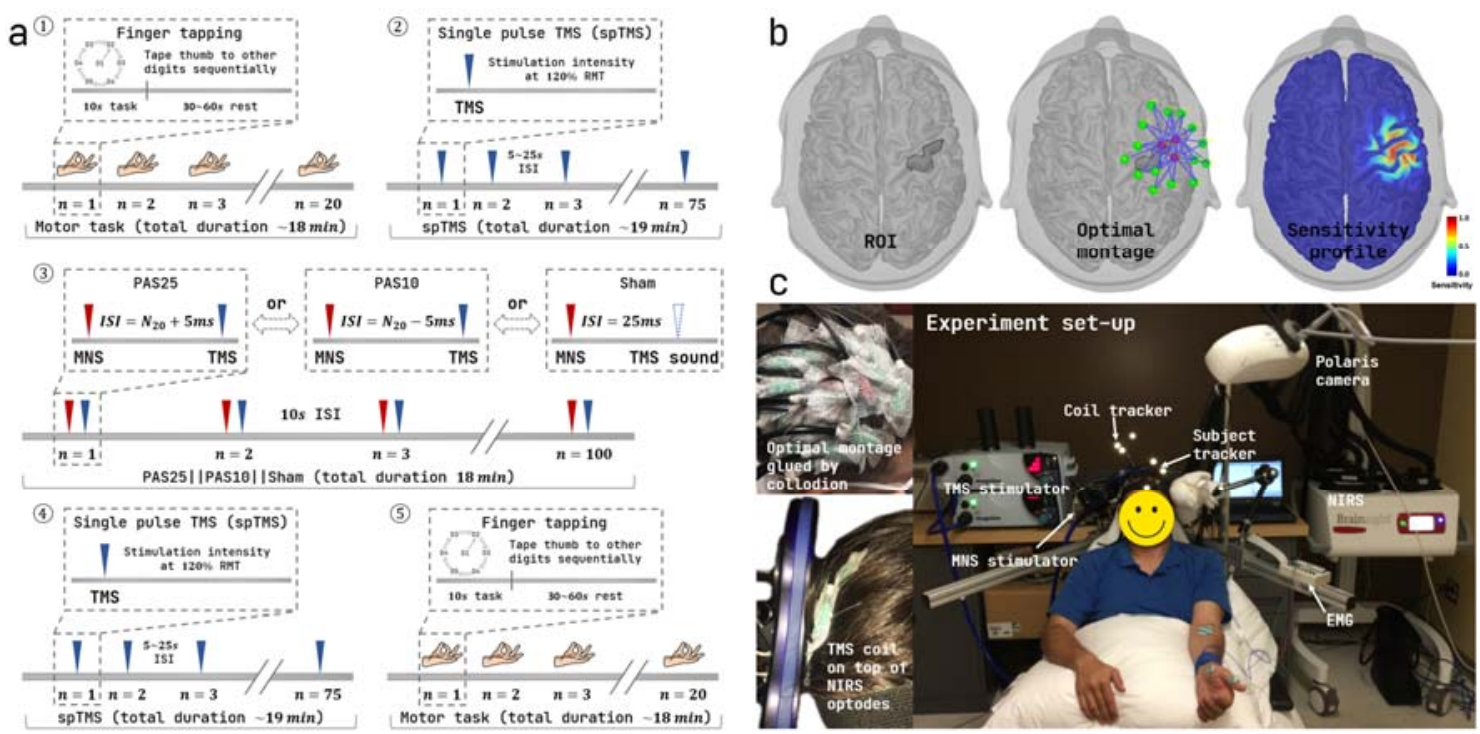

312 Fig.1. Experimental paradigm and set-up. a) experiment paradigm ordered by time: 1) is a block designed finger-tapping task consisted of 20 blocks, each contained $10 \mathrm{~s}$ task and $30 \mathrm{~s}$ to $60 \mathrm{~s}$ rest; subjects were informed to tapping their left thumb to the other 4 digits sequentially at around $2 \mathrm{~Hz} ; 2$ ) is an event-related designed single pulse TMS (spTMS) run consisted of 75 events jittered from $5 \mathrm{~s}$ to 25s. 3) PAS25/PAS10/sham-PAS consisted of 100 pairs of stimulations, interleaved by 10s; 4) and 5) repeated 2) and 1), respectively, after the PAS intervention. b) personalized optimal montage for fNIRS acquisition. 3 sources (red dots) and 15 detectors (green dots) were selected to optimize the sensitivity of fNIRS montage to a predefined ROI, the right MI hand knob (outlined using a black profile) along the cortical surface. c) an overview of the experimental set-up, the personalized optimal montage was glued on the scalp using clinical adhesive - collodion; TMS coil was placed on top of the fNIRS optodes to target the 'hot spot' which corresponded to subject's left thumb, note that the low-profile feature of the fNIRS optodes allowed less TMS intensity decreases when departing from

324 the scalp surface; a neuro-navigation system was used to guild the placement of the TMS coil and the digitization of the fNIRS optodes.

\subsection{Data preprocessing}

\subsubsection{EMG data processing}

328 EMG data collected during spTMS runs were processed using Brainstorm software (Tadel et 329 al., 2011) (https://neuroimage.usc.edu/brainstorm/) to extract MEP amplitudes. Raw EMG 330 data were first band-pass filtered between 3 and $2000 \mathrm{~Hz}$. A time window from $-10 \mathrm{~ms}$ to 331 100ms around the stimulation onset was defined to extract MEP trials. These trials were then 
332 baseline corrected (-10ms to $0 \mathrm{~ms})$, and the peak-to-peak amplitude of each MEP trial was

333 calculated. Note that throughout the analysis reported in this study, none of the single MEP

334 trials was excluded to preserve the intrinsic variability of MEP peak-to-peak amplitude

335 measures. Hereby, for convenience, we will denote as "MEP", the actual MEP peak-to-peak

336 amplitude, as often considered in TMS literature.

337 The output of the whole EMG data preprocessing section was a set of 75 MEPs estimated for 338 each participant (specified by subject ID from 1 to 16), each intervention (PAS25, PAS10 or 339 sham) and time (pre-PAS or post-PAS).

\section{$340 \quad$ 2.3.2 fNIRS data processing}

341 fNIRS data processing was performed using the open-source fNIRS processing plugin 342 NIRSTORM (https://github.com/Nirstorm/nirstorm) implemented in our lab in Brainstorm 343 software (Tadel et al., 2011) (https://neuroimage.usc.edu/brainstorm/). Raw fNIRS data were 344 first preprocessed following standard recommendations (Yücel et al., 2020) and then 345 converted to optical density changes (i.e., $\Delta \mathrm{OD}$ ). For each task run, $20 \Delta \mathrm{OD}$ epochs were 346 extracted within a time window ranging from -10 s to 30 s around task onsets. To reduce 347 motion artifacts and obtain the distribution of averaged $\triangle \mathrm{OD}$ epochs for each run, we sub348 averaged 16 out of $20 \Delta \mathrm{OD}$ epochs for all possible unique combinations (i.e., $C_{20}^{16}=4845$ 349 possibilities) and selected 50 of these sub-averaged $\triangle \mathrm{OD}$ epochs below and above the median 350 of the signal to noise ratio (SNR). To obtain the distribution of spatiotemporal map of $351 \mathrm{HbO} / \mathrm{HbR}$ responses for each finger-tapping run along the cortical surface, we applied 3D

352 fNIRS reconstruction workflow (Cai et al., 2021c) using personalized optimal montage and 353 maximum entropy on the mean (MEM) to these 101 sub-averaged $\triangle \mathrm{ODs}$. $\mathrm{HbO} / \mathrm{HbR}$ 354 spatiotemporal maps of each subject during each finger-tapping run (e.g., $101 \mathrm{HbO}$ maps for 355 Sub01 during pre-PAS25 finger-tapping) were co-registered to the mid-surface of the MNI 356 ICBM152 template (Fonov et al., 2009, 2011), using FreeSurfer spherical transformation. An 357 ROI was defined along the template surface as the "hand knob", to cover the cortical regions 358 that control finger tapping. Finally, reconstructed $\mathrm{HbO} / \mathrm{HbR}$ time courses (0s to 30s) within 359 this "hand knob" ROI were averaged to represent the hemodynamic responses of each 360 specific finger-tapping run. The output of the whole fNIRS data preprocessing section was a 361 set of 80 runs (i.e., 40 sessions (16 PAS25+12 PAS10+12 sham) $\times 2$ times) of 101 362 reconstructed $\mathrm{HbO} / \mathrm{HbR}$ time course, for each run specified by subject (ID 1 to 16), 363 intervention (PAS25, PAS10 or sham) and time (pre-PAS or post-PAS). Please see further 364 details of fNIRS data processing in Appendix 1 and our previous study (Cai et al., 2021b). 


\section{$365 \quad 2.4$ Hierarchical Bayesian Modeling}

366 To clarify the notation used in the following model equations, we used small letters to denote 367 a scalar variable (e.g., $\mu$ for the mean of a Gaussian distribution) and capital letters to denote

368 a matrix (e.g., $\Sigma$ for the covariance matrix of a multivariate Gaussian distribution). A list of 369 scalar values from one specific variable is represented by a small letter along with a subscript 370 letter, for instance, a symbol $\mu_{s}$ refers to a list of means, and the subscript $s$ represents each 371 individual element of this list (mean for the $s^{\text {th }}$ session). The dimensionality of each list is 372 given by the range of $s$ (e.g., $s=1,2,3, \ldots 40$, for $s^{\text {th }}$ session). If subscript letter(s) is 373 contained in square brackets, it means that the individual element of this list variable is 374 differentiated by the model using index variables. Such as, $i$ in intercept $_{[i=1,2,3]}$ indicates 375 our model differentiates the intercept parameter for each intervention type by index variable $376 i=1,2,3,1$ for PAS25, 2 for PAS10 and 3 for sham.

\section{2.4.1 Hierarchical Bayesian Model \#1: Assessment of PAS effects on cortical excitability}

378 We proposed a hierarchical Bayesian model to assess PAS effects on M1 cortical excitability, 379 which was evaluated using the MEPs measured during spTMS runs before (pre-) and after 380 (post-) each PAS intervention. This model consists of two parts: 1) a measurement error 381 model taking into account the variability of MEPs within each spTMS run and 2) a 382 multivariate hierarchical model describing post-intervention MEP as a linear function of pre383 intervention MEP.

\section{1) A model of measurement error}

385 We assume the "empirical" mean of the observed MEP in each run to be drawn from a 386 Gaussian distribution with the mean equals to the 'true' MEP amplitude and the scale equals 387 to the standard error of all MEPs trials. The 'true' and observed ('obs') MEP of the pre-PAS 388 spTMS run can be expressed as follows,

$$
\begin{aligned}
M E P_{\text {obs }, s}^{\text {pre }} & \sim \mathcal{N} \text { ormal }\left(M E P_{\text {true }, s}^{\text {pre }}, M_{\text {S }} P_{\text {se }, s}^{\text {pre }}\right) \\
M E P_{\text {true }, s}^{\text {pre }} & \sim \mathcal{N} \text { ormal }(0.5,1) \\
s & =1,2,3, \ldots 40, \text { for } s^{\text {th }} \text { session }
\end{aligned}
$$

389 where $M E P_{\text {true }, S}^{\text {pre }}$ is the 'true' value of the pre-PAS mean MEP for session $S . M E P_{o b s, S}^{\text {pre }}$ is the "empirical" mean of the observed MEP from the same run expressed as, 


$$
\begin{aligned}
M E P_{o b s, s}^{p r e} & =\frac{\sum_{k=1}^{N} M E P_{k, s}^{p r e}}{N} \\
k & =1,2,3, \ldots 75, \text { for } k^{\text {th }} \text { trial }
\end{aligned}
$$

391 where $M E P_{k, s}^{p r e}$ represents the pre-PAS MEP of the $k^{\text {th }}$ trial from a total of $\mathrm{N}=75$ trials in 392 session $s$. The corresponding measurement error $M E P_{s e, s}^{p r e}$ is then represented by the standard 393 error of the MEP among all 75 trials, estimated by,

$$
M E P_{s e, s}^{p r e}=\sqrt{\frac{\sum_{k=1}^{N}\left(M E P_{k, s}^{p r e}-M E P_{o b s, s}^{p r e}\right)^{2}}{N(N-1)}}
$$

394 Finally, substituting (2) and (3) into (1), both empirical mean and variance estimated over the 39575 observed pre-PAS MEPs of a specific session were modeled to estimate the 'true' 396 corresponding amplitude. For the prior distribution of $M E P_{t r u e, s}^{\text {pre }}$, we applied a weakly 397 informed prior (Gelman et al., 2008, 2017; Gabry et al., 2019) consisting in a Gaussian 398 distribution $\mathcal{N}$ ormal $(0.5,1)$. Note that all observed MEPs $\left(M E P_{o b s, s}^{\text {pre,post }}\right.$ and $M E P_{s e, s}^{\text {pre,post }}$, 399 for 40 Sessions $\times 2$ Times $($ pre $-/$ post $-P A S)=80)$ were normalized by the global 400 maximum value of $M E P_{o b s, s}^{\text {pre,post }}$ to ensure a $[0,1]$ range. Then, 0.5 appears as an appropriate 401 prior of the mean when nothing is known about the MEP amplitude, but only the range (i.e., $402(0+1) / 2=0.5)$.

403 The 'true' MEP in the post-PAS spTMS run was then modeled as follows,

$$
\begin{aligned}
M E P_{o b s, s}^{\text {post }} & \sim \mathcal{N} \text { ormal }\left(M E P_{\text {true }, s}^{\text {post }}, M E P_{s e, s}^{\text {post }}\right) \\
M E P_{o b s, s}^{\text {post }} & =\frac{\sum_{k=1}^{N} M E P_{k, s}^{\text {post }}}{N} \\
M E P_{s e, s}^{\text {post }} & =\sqrt{\frac{\sum_{k=1}^{N}\left(M E P_{k, s}^{\text {post }}-M E P_{o b s, s}^{\text {post }}\right)^{2}}{N(N-1)}}
\end{aligned}
$$

404 Note that the prior distribution of the parameter $M E P_{\text {true }, s}^{\text {post }}$ is defined in the next section, 405 within the context of hierarchical multivariate linear regression. 
407 PAS effects on M1 cortical excitability were then modeled using a multivariate linear 408 regression model, in which $M E P_{\text {true,s }}^{\text {post }}$ and $M E P_{\text {true,s }}^{\text {pre }}$ were considered as the dependent and 409 predictor variables, respectively.

$$
\begin{gathered}
M E P_{\text {true }, s}^{\text {post }} \sim \mathcal{N} \operatorname{dormal}\left(\mu_{s}, \sigma\right) \\
\mu_{s}=\text { intercept }_{[i]}+\left(\operatorname{group}_{[i]}+\operatorname{actor}_{[a, i]}\right) \cdot M E P_{\text {true }, s}^{\text {pre }} \\
i=1,2 \text { and } 3, \text { for } i^{\text {th }} \text { intervention } \\
a=1,2,3, \ldots 16, \text { for } a^{\text {th }} \text { subject } \\
s=1,2,3, \ldots 40, \text { for } s^{\text {th }} \text { session }
\end{gathered}
$$

410 where $\mu_{s}$ is the mean of $M E P_{\text {true }, s}^{\text {post }}$, predicted by $M E P_{\text {true,s }}^{\text {pre }}$ using the following linear model: $411 \mu_{s}=$ intercept $_{[i]}+\left(\operatorname{group}_{[i]}+\operatorname{actor}_{[a, i]}\right) \cdot M E P_{\text {true }, s}^{\text {pre }}$, and $\sigma$ is the error of the linear 412 regression (i.e., the scale of the normal distribution). We added the following index variables 413 to differentiate subject, intervention (PAS25/PAS10/sham) and time (pre-/post-PAS) in the 414 model. intercept $t_{[i]}$ is the intercept of the linear regression, for the $i^{\text {th }}$ intervention, in 415 which $i=1,2$ and 3 refers to PAS25, PAS10 and sham, respectively. The slope parameter 416 is modeled using two parts, a group-level slope parameter group $_{[i]}$, specific for each 417 intervention $i$, and a parameter modeling inter-subject variability, denoted as actor $_{[a, i]}$, for 418 each intervention $i$ and each subject $a$, associated with the following prior model:

$$
\begin{aligned}
& {\left[\begin{array}{l}
\operatorname{actor}_{[a, i=1]} \\
\operatorname{actor}_{[a, i=2]} \\
\operatorname{actor}_{[a, i=3]}
\end{array}\right] \sim \mathcal{M} \text { ultiNormal }\left(\left[\begin{array}{l}
0 \\
0 \\
0
\end{array}\right], \Sigma\right)} \\
& \Sigma=\Sigma_{\text {actor }} \cdot R h o \cdot \Sigma_{\text {actor }} \\
& =\left(\begin{array}{ccc}
\sigma_{1} & 0 & 0 \\
0 & \sigma_{2} & 0 \\
0 & 0 & \sigma_{3}
\end{array}\right)\left(\begin{array}{ccc}
1 & \rho_{12} & \rho_{13} \\
\rho_{21} & 1 & \rho_{23} \\
\rho_{31} & \rho_{32} & 1
\end{array}\right)\left(\begin{array}{ccc}
\sigma_{1} & 0 & 0 \\
0 & \sigma_{2} & 0 \\
0 & 0 & \sigma_{3}
\end{array}\right)
\end{aligned}
$$

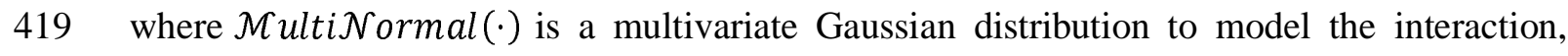
420 which allows the effects of each specific intervention to vary for each subject, meaning each 421 subject can respond to each intervention differently. We defined this multivariate Gaussian 422 prior distribution to have zero means ( 3 elements vector) therefore assuming all the subjects 423 to have zero mean deviation around the group-level slope parameter group $_{[i]}$. Rho and $424 \Sigma_{\text {actor }}$ denote respectively the correlation matrix and the scale matrix of the covariance 425 matrix $\Sigma$ of the multivariate Gaussian distribution. $\sigma_{1,2,3}$ is the scale among all subjects within 
426 each intervention group, for example, $\sigma_{3}$ is the scale of the vector actor $_{[a, i=3]}$ for sham. $\rho$ is

427 the correlation between pair-wised interventions, for instance, $\rho_{12}$ represents the correlation

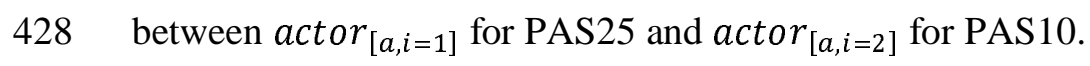

429 Weakly informed priors were assigned to the parameters in (5) and (6) as follows,

$$
\begin{gathered}
\text { intercept }_{[i]} \sim \mathcal{N} \operatorname{ormal}(0,0.1) \\
\operatorname{group}_{[i]} \sim \log \mathcal{N} \operatorname{ormal}(\log (1), 0.5) \\
\rho \sim \mathcal{L} \mathcal{K} \mathcal{J} \operatorname{corr}(2) \\
\sigma \sim \mathcal{H} \text { alf } \mathcal{N} \operatorname{ormal}(0,1)
\end{gathered}
$$

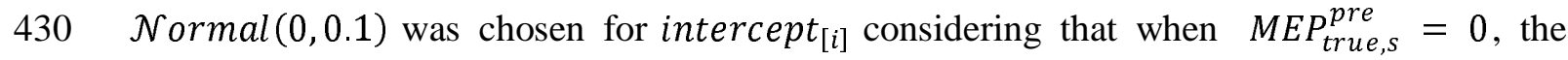
431 corresponding $M E P_{\text {true }, \text { sos }}^{\text {post }}$ should not be too much apart from $0 . \log \mathcal{N} \operatorname{ormal}(\log (1), 0.5)$ 432 was selected for the group-level slope to ensure it is a positive value with a median of 1. 433 Therefore, without knowing any intervention type, the slope should be equal to 1, assuming 434 there is no averaged PAS effect among subjects when the intervention type is not known. $435 \mathcal{L} \mathcal{K} \mathcal{J} \operatorname{corr}(\eta=2)$, the Lewandowski-Kurowicka-Joe distribution (Lewandowski et al., 436 2009), is a weakly informative prior for the correlation parameter $\rho$ that does not prioritize 437 extreme correlation values such as \pm 1 , where $\eta$ is a positive parameter. $\eta=1$ denotes 438 uniform density of $\rho$. The larger $\eta$ is (when compared to 1), the least likely the extreme 439 correlation values to occur (sharper probability density distribution). We selected $\eta=2$ as a 440 weakly informed prior commonly considered in Bayesian data analysis (McElreath, 2020). 441 Finally, $\mathcal{H}$ alf $\mathcal{N} \operatorname{ormal}(0,1)$ was used for variance parameters to ensure a positive value, 442 whereas its likelihood decreased following the positive half of a $\mathcal{N} \operatorname{ormal}(0,1)$ distribution, 443 when variance increases. As denoted previously, we normalized all data $M E P_{k, s}^{\text {pre }}$ and $444 M E P_{k, s}^{p o s t}$ within the range $[0,1]$, therefore $\mathcal{N} \operatorname{ormal}(0,1)$ is considered as a conservative 445 ("flat") enough prior, not reducing the variance. 


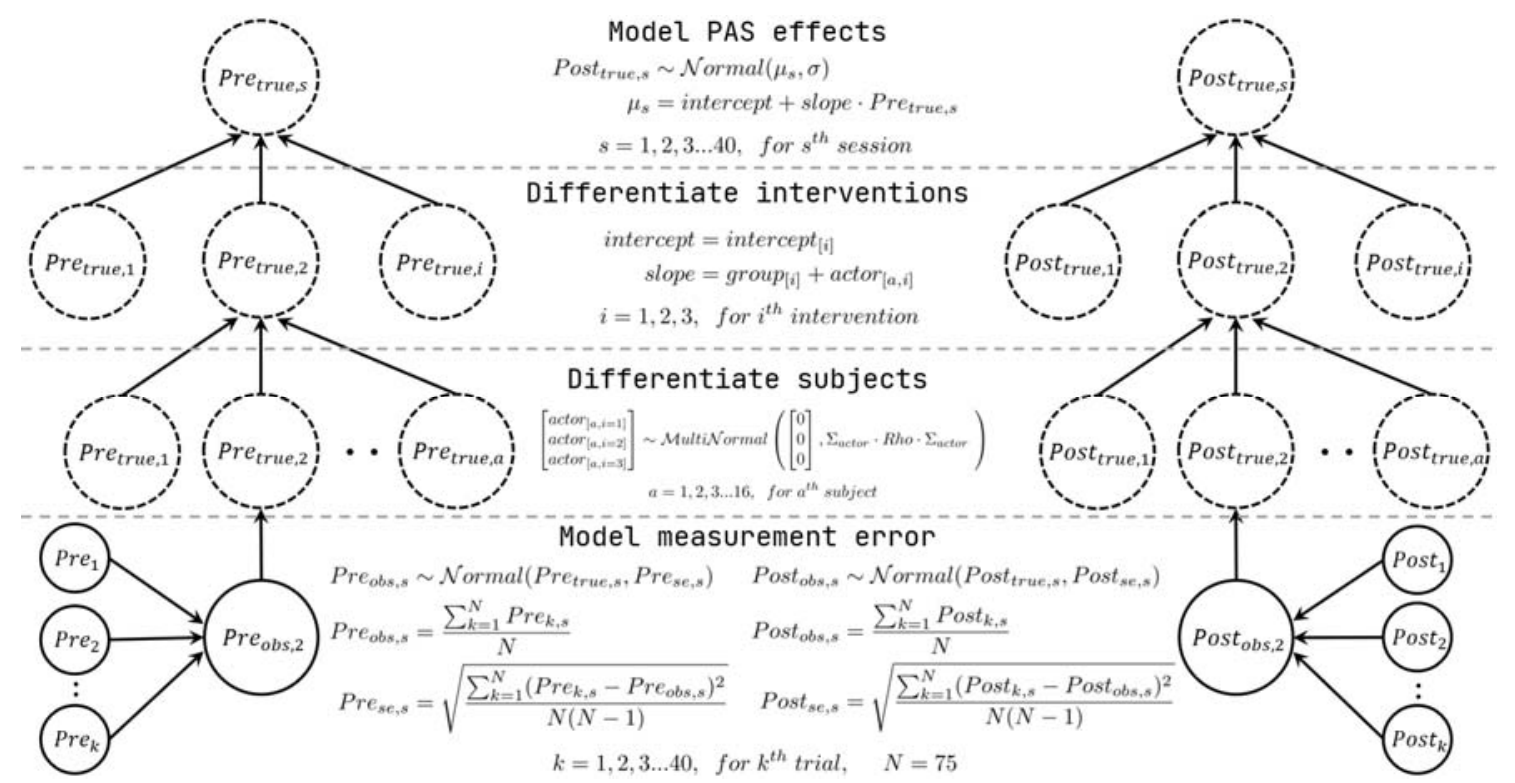

Fig.2. The hierarchical model of PAS effects on either cortical excitability (MEP) or hemodynamic

responses $(\boldsymbol{H b})$. From bottom to top, 1) a measurement error model assuming the mean of the variable of interest (either the observed MEPs, e.g., $M E P_{s e, s}^{p r e}$; or a spline weight of $\mathrm{HbO/HbR}$ time course, e.g., $w_{o b s, s, n}^{\text {pre }}$ of each run at a different time (pre-/post-) is drawn from a Gaussian distribution. The mean of this Gaussian distribution is the 'true' value of the variable of interest, and the scale is the corresponding standard error; 2) each subject and intervention were differentiated using index variables; 3) PAS effects were modeled by linear regression in which the 'true' postvariable of interest was predicted by the 'true' pre-variable of interest. Solving this hierarchical model by Bayesian allows partial pooling on each parameter to reduce the uncertainty.

\subsubsection{Hierarchical Bayesian Model \#2: Assessment of PAS effects on task-related} $\mathrm{HbO} / \mathrm{HbR}$

A similar hierarchical multivariate linear regression model is proposed to assess PAS effects on task-related fNIRS hemodynamic responses. This model is very similar to previous Model\#1, the main difference being that the input variables to the model are now 'features' representing hemodynamic responses during the finger-tapping task. Instead of extracting amplitudes at a particular time sample of $\mathrm{HbO} / \mathrm{HbR}$ time courses, for instance, the hemodynamic peak amplitude, or performing a local average within a specific time window, in this Model \#2, we conducted a procedure to model the PAS effects over the whole time course of $\mathrm{HbO} / \mathrm{HbR}$ responses to finger tapping. 
468 selected time range [0s, 30s]. To lower the dimension of the input to the model, resulting time 469 courses were then projected on B-splines temporal basis functions (Boor, 2001; Gelman et 470 al., 2013; Hastie, 2017). Therefore, hemodynamic responses were expressed as a weighted 471 linear combination of those basis functions, considering the hierarchical model summarized 472 in Fig.3. Please note that $\mathrm{Hb}$ refers to either $\mathrm{HbO}$ or $\mathrm{HbR}$ in the model, which was fitted 473 separately for each chromophore,

$$
\begin{aligned}
H b_{o b s, s}^{\text {pre }}(t) & \sim \mathcal{N} \text { ormal }\left(\mu_{s}^{\text {pre }}(t), H b_{s d, s}^{\text {pre }}(t)\right) \\
\mu_{s}^{\text {pre }}(t) & =w_{o b s, s, n}^{\text {pre }} \times B_{n}(t) \\
w_{o b s, s, n}^{\text {pre }} & \sim \mathcal{N} \operatorname{Normal}(0,10) \\
H b_{o b s, s}^{\text {post }}(t) & \sim \mathcal{N} \operatorname{ormal}\left(\mu_{s}^{\text {post }}(t), H b_{s d, s}^{\text {post }}(t)\right) \\
\mu_{s}^{\text {post }}(t) & =w_{\text {obs }, s, n}^{\text {post }} \times B_{n}(t) \\
w_{o b s, s, n}^{\text {post }} & \sim \mathcal{N} \text { ormal }(0,10) \\
n & =1,2,3, \ldots 10, \text { for } n^{\text {th }} \text { weight } \\
t & =0 s \text { to } 30 \text { s with a step of } 0.5 s \\
s & =1,2,3, \ldots 40, \text { for } s^{\text {th }} \text { session }
\end{aligned}
$$

474 where $H b_{o b s, S}^{\text {pre }}(t)$ is the observed empirical mean of pre-PAS $\mathrm{HbO} / \mathrm{HbR}$ responses among all 475101 sub-averaged time courses, for a specific finger-tapping run (e.g., finger-tapping run in 476 pre-PAS25 of Sub01) of a specific session $s$ at a specific time point $t$. H $b_{o b s, S}^{\text {pre }}(t)$ is assumed to follow a Gaussian distribution with a mean of $\mu_{s}^{\text {pre }}(t)$ and a scale of $H b_{s d, s}^{\text {pre }}(t)$, where $478 H b_{s d, s}^{p r e}(t)$ is the corresponding standard deviation estimated among all 101 sub-averaged time courses. Note that all pre- and post-PAS $\mathrm{HbO} / \mathrm{HbR}$ time courses in one session were normalized by the global maximum amplitude to be within the range $[-1,1]$. Then, $\mu_{s}^{\text {pre }}(t)$ representing the mean time course of the true pre-PAS $\mathrm{HbR} / \mathrm{HbO}$ for time sample $t$ and session $\mathrm{s}$, was defined as a linear combination of $n=10 \mathrm{~B}$-spline basis functions $B_{n}(t)$ using the corresponding weight $w_{o b s, s, n}^{\text {pre }}$. Each basis function $B_{n}(t)$ was defined as a $3^{\text {rd }}$ order polynomial function. A similar model structure was applied to data and parameters corresponding to the post-PAS finger-tapping run. To model the temporal response using Bspline, we selected 10 knots pivoted at the percentiles of time sequence $t=0$ s to $30 \mathrm{~s}$ with a step of $0.5 s$; therefore, 10 corresponding weights and basis functions, as illustrated in the second column of Fig.3. Using this Bayesian spline model, not only the averaged time course of $\mathrm{HbO} / \mathrm{HbR}$, but also their corresponding standard deviation over the 101 sub-averaged, for 
490 each time point, were projected in the 'spline space'. This means the averaged time course of $491 \mathrm{HbO} / \mathrm{HbR}$ can be recovered by the linear combination of the mean of each weight (over 101 492 sub-averaged) and basis functions $B_{n}(t)$, whereas the standard deviation of $\mathrm{HbO} / \mathrm{HbR}$ time 493 course is reflected by the linear combination of the standard deviation of each weight (over 494101 sub-averaged) and $B_{n}(t)$. Note that the use of spline basis functions in this study was 495 mainly to reduce the dimensionality of the $\mathrm{HbO} / \mathrm{HbR}$ time course from 60 sampling points to 49610 weights, while preserving the variability structure to be modeled. Therefore, selecting 10 497 spline knots was a trade-off between: 1) choosing fewer knots that would result in eventual 498 distortion of the $\mathrm{HbO} / \mathrm{HbR}$ time courses, involving too much temporal smoothness; 2) adding more knots that would increase the dimensionality of the data after projection. Hence, our empirical choice ensured accurate representation of the whole $\mathrm{HbO} / \mathrm{HbR}$ time courses with a 501 minimum dimensionality span. Importantly, projecting to spline space also preserved the 502 autocorrelation of the $\mathrm{HbO} / \mathrm{HbR}$ time courses per se, which could not be achieved when 503 simply applying the same hierarchical model on each of 60 data points independently. 504 Finally, $\mathcal{N}$ ormal $(0,10)$ is considered as a weakly informative prior for spline weight 505 considering the $\mathrm{HbO} / \mathrm{HbR}$ time course was normalized within the range $[-1,1]$.

506 We then embedded this spline model of the hemodynamic response within the same 507 hierarchical model proposed in the previous section (Model\#1), for instance, replacing the $508 M E P_{o b s, s}^{p r e}$ with the spline weights $w_{o b s, s, n}^{p r e}$ as follows, also illustrated in the third column of 509 Fig.3.

$$
\begin{aligned}
w_{\text {obs }, s, n}^{\text {post }} & \sim \mathcal{N} \operatorname{ormal}\left(w_{\text {true }, s, n}^{\text {post }}, w_{s d, s, n}^{\text {post }}\right) \\
w_{\text {obs }, s, n}^{\text {pre }} & \sim \mathcal{N} \operatorname{ormal}\left(w_{\text {true }, s, n}^{\text {pre }}, w_{s d, s, n}^{\text {pre }}\right) \\
w_{\text {true }, s, n}^{\text {post }} & \sim \mathcal{N} \operatorname{ormal}\left(\mu_{s}, \sigma\right) \\
\mu_{s} & =\operatorname{intercept}_{[i]}+\left(\operatorname{group}_{[i]}+\operatorname{actor}_{[a, i]}\right) \cdot w_{\text {true }, s, n}^{\text {pre }} \\
w_{\text {true }, s, n}^{\text {pre }} & \sim \mathcal{N} \operatorname{dormal}(0,10)
\end{aligned}
$$

510 where $w_{o b s, s, n}^{\text {post }} w_{o b s, s, n}^{\text {pre }}, w_{s d, s, n}^{\text {post }}$ and $w_{s d, s, n}^{\text {pre }}$ were all calculated from the corresponding 511 posterior of spline weights estimated from equation (8). In total, 10 models were considered 512 for 10 pairs of weights (pre- and post-PAS) to encode the PAS effects for either $\mathrm{HbO}$ or $\mathrm{HbR}$ 513 separately. $w_{o b s, s, n}^{\text {post }}$ and $w_{o b s, s, n}^{\text {pre }}$ are referring to the empirical mean of each spline weight for 514 either pre- or post-PAS $\mathrm{HbO} / \mathrm{HbR}$ for session $s$, estimated from the corresponding posterior 515 of spline weights in equation (8). The scale of Gaussian distribution in the measurement error 
516 model was then $w_{s d, s, n}^{p o s t}$ and $w_{s d, s, n}^{\text {pre }}$, respectively. Note that equation (8) resulted in the

517 estimated posterior distribution of $w_{o b s, s, n}^{\text {post }}$ and $w_{o b s, s, n}^{\text {pre }}$ after projecting $\mathrm{HbO}$ or $\mathrm{HbR}$ time

518 course to the spline space, then scales of Gaussian distributions used for the measurement

519 error model in (9) were directly reflected by the standard deviation of the posterior

520 distribution (denoted as $s d$ in subscript). Finally, the intervention, subject index variables,

521 and priors to be considered for this model, were similar to those previously introduced for

522 Model\#1, so the PAS effects on $\mathrm{HbO} / \mathrm{HbR}$ whole time course were then encapsulated in the

523 hierarchical model of spline weights.
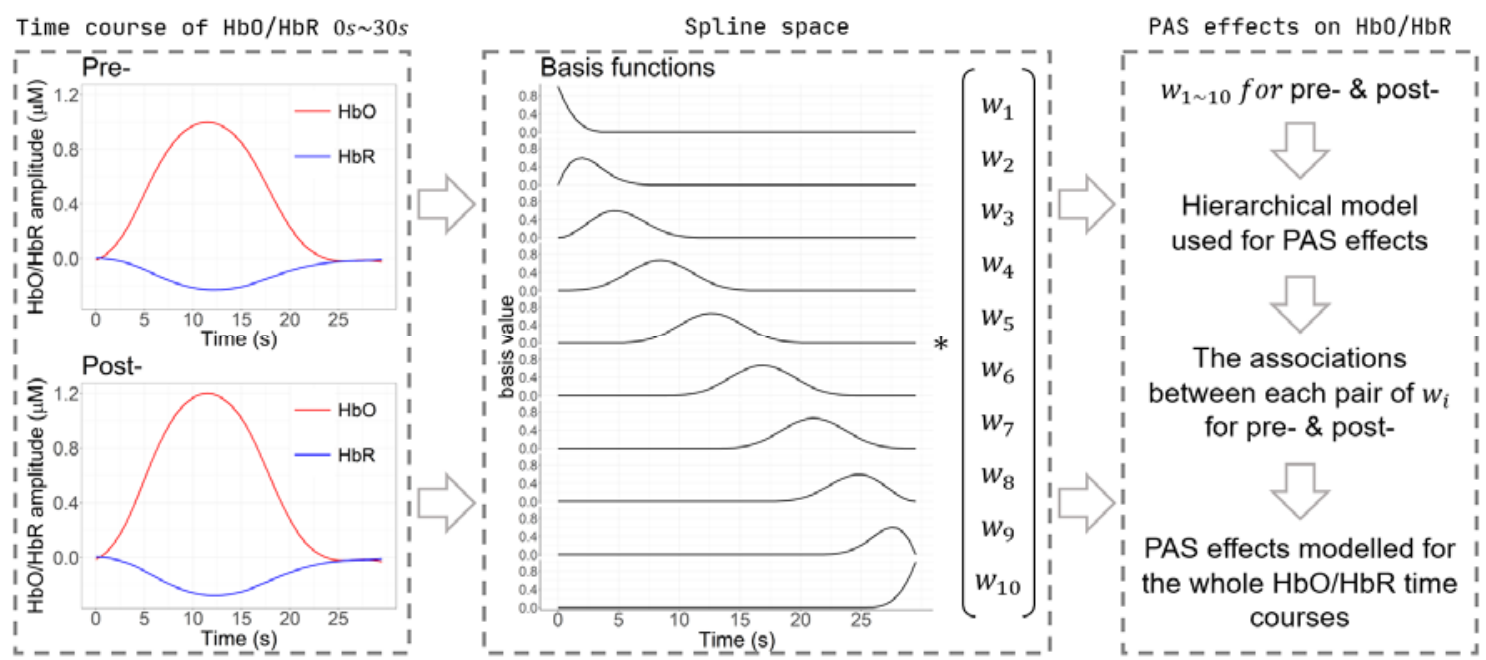

525 Fig.3. The hierarchical model for PAS effects on the whole HbO/HbR time course. HbO/HbR time

526 courses, from 0s to 30s, before and after each intervention session were selected as the inputs of the

527 model. They were projected into the spline space which is composed of 10 predefined basis functions

528 ( $3^{\text {rd }}$ order polynomial with 10 knots). The linear combination of basis function using 10

529 corresponding weights $w_{1 \sim 10}$ can fully recover the $\mathrm{HbO/HbR}$ time course. The resulting pre-and

530 post-PAS spline weights were then fed into the hierarchical model, similar to model \#1 to estimate the

531 PAS effects on each weight. Therefore, the associations between each pair of weights encapsulated

532 the PAS effects on the whole time course of $\mathrm{HbO} / \mathrm{HbR}$.

533 2.4.3 Hierarchical Bayesian Model \#3: Relationship between PAS effects on task534 related M1 hemodynamic activity and PAS effects on M1 excitability

535 In this third model, we propose to investigate the interactions between 1) PAS effects on M1 536 excitability (PAS effects on MEP, represented by the slope parameter in Model\#1), and 2) 537 PAS effects on reconstructed hemodynamic finger tapping responses (PAS effects on $538 \mathrm{HbO} / \mathrm{HbR}$, represented by the slope parameter in Model\#2 for a specific weight $\left.w_{n}\right)$. We 
539 assumed the relationship between task-related M1 hemodynamic activity and M1 excitability

540 was not intervention specific, then the previous hierarchical models (i.e., Model\#1 and

541 Model\#2) were modified to be only session specific, i.e., only index variable of session $s$ was

542 used, whereas intervention and subject index $i$ and $a$ were ignored.

$$
\begin{aligned}
& {\left[\begin{array}{c}
\operatorname{slope}_{[s]}^{M E P} \\
\operatorname{slope}_{[s]}^{H b}
\end{array}\right] \sim \mathcal{M} \text { ultiNormal }\left(\left[\begin{array}{l}
0 \\
0
\end{array}\right], \Sigma_{\text {slope }}\right)} \\
& \Sigma_{\text {slope }}=\Sigma_{\text {session }} \cdot R h o_{\text {slope }} \cdot \Sigma_{\text {session }} \\
& =\left(\begin{array}{cc}
\sigma_{M E P} & 0 \\
0 & \sigma_{H b}
\end{array}\right)\left(\begin{array}{cc}
1 & \rho_{M E P-H b} \\
\rho_{H b-M E P} & 1
\end{array}\right)\left(\begin{array}{cc}
\sigma_{M E P} & 0 \\
0 & \sigma_{H b}
\end{array}\right) \\
& s=1,2,3, \ldots 40 \text {, for } s^{\text {th }} \text { session }
\end{aligned}
$$

543 The interactions were modeled using a multinormal distribution, in which the parameter $544 \rho_{M E P-H b}$ in the $R h o_{\text {slope }}$ matrix denotes the correlation between the two slopes (representing 545 the PAS effects in both linear models). The same model was fitted separately when 546 investigating either the relationship between MEP and $\mathrm{HbO}$ or between MEP and HbR. $547 \operatorname{slope}_{[s]}^{M E P}$ is the session specific slope parameter in Model\#1. Similarly, slope $e_{[s]}^{H b}$ (either HbO 548 or $\mathrm{HbR}$ ) is the session specific slope parameter in Model\#2 for one of the corresponding 549 spline weights $w_{1 \sim 10} . \sigma_{M E P}$ and $\sigma_{H b}$ are the standard deviations of $\operatorname{slope}_{[s]}^{M E P}$ or $\operatorname{slope} e_{[s]}^{H b}$, 550 respectively. Note that this model was fitted for each spline weight separately - 10 correlation 551 investigations between MEP and each spline weight were conducted. Therefore, the posterior 552 distribution $\rho_{M E P-H b}$ inferred for a specific spline weight can be interpreted as the 553 correlation between brain excitability and task-related cortical hemodynamic activity at a 554 specific period (e.g., $w_{5}$ reflecting $\mathrm{HbO} / \mathrm{HbR}$ fluctuations around the peak time point of the 555 hemodynamic response). For the parameters of the Multinormal distribution, we considered 556 the same weakly informed priors as those proposed in Model\#1 and Model\#2. 


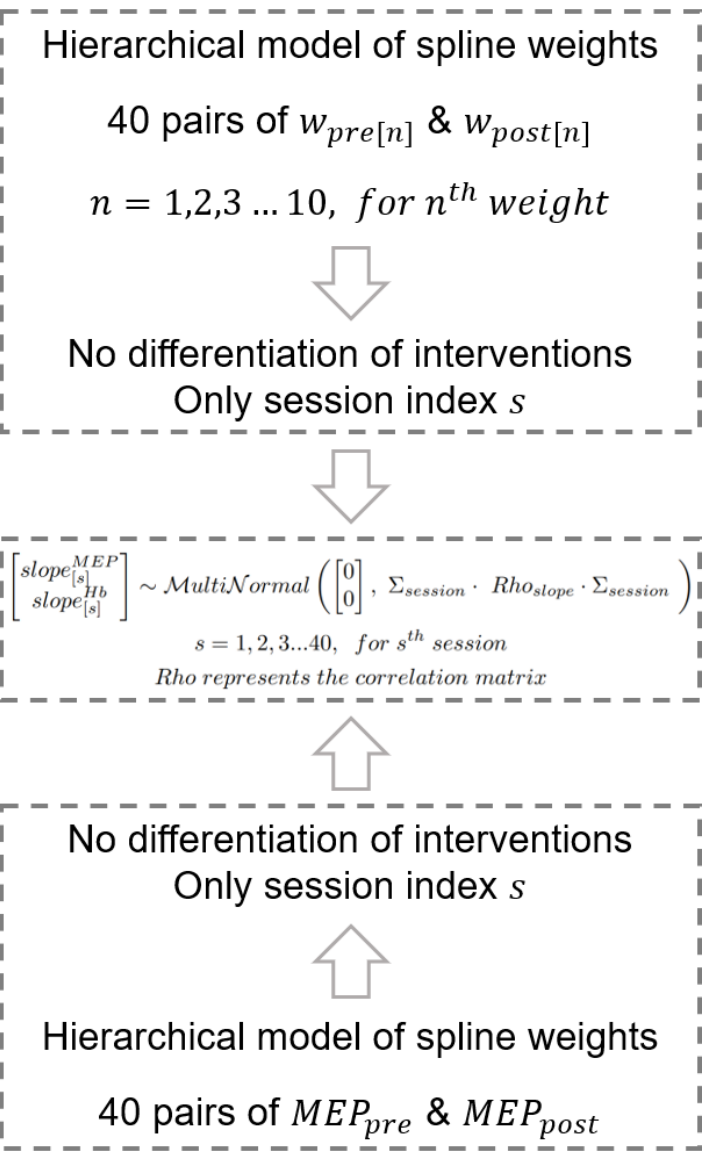

558 Fig.4. Modeling the relationship of task-related M1 hemodynamic activity and M1 excitability. For

559 MEP, which represented the M1 excitability, the previous Model \#1 was modified to be session-

560 specific only. For spline weights, which represented the features of task-related HbO/HbR time

561 course, the previous 'Model \#2 was also modified to be session-specific only. The association between

562 the slope $e_{M E P}$ in MEP model and slope ${ }_{H b}$ represented by any of the spline weight of the HbO/HbR

563 time course model were described by a multinormal distribution.

\section{$564 \quad 2.5$ Prior predictive simulation}

565 To justify the choices of 'weakly informed' prior, a prior predictive simulation was

566 conducted in Fig.3. The prior predictive simulation consists in a generative process simply

567 checking what kind of data we would expect to generate from our hierarchical models, when

568 applying all possible values of the parameters considering the proposed prior distributions of

569 the model. Then by comparing the distribution of data generated by our model, to the domain

570 knowledge, one can assess whether the proposed priors could be overregulating or not 571 objective (e.g., too strongly informed). In our study, PAS effects were modeled using linear 572 regression. To perform prior predictive simulation, we considered prior predictive simulation 573 to draw 1000 lines following the prior distributions of the intercept and slope in the 
574 normalized pre-MEP vs. post-MEP amplitude plane. Then the distribution of generated

575 regression lines was compared to three reference lines summarizing our knowledge of the

576 problem. In detail, these three reference lines were featuring a slope of $0.2,1$ and 3 ,

577 respectively and an intercept of 0 . When the intercept is set to 0 , the slope just refers to the

578 ratio of post- over pre-PAS MEP amplitude, which was used in conventional analysis to

579 represent the PAS effects. Whereas a slope of 1 would then correspond to no effect (post-

580 /pre-PAS ratio of 1), the reference slopes of 0.2 and 3 represented the thresholds for outliers

581 of extremely small or large MEP ratios reported in Kriváneková et al., 2013.

\section{$582 \quad$ 2.6 Hierarchical Bayesian model fitting}

583 In this study, we used the R Version 4.0.3 (R Core Team, 2020) distribution of the Stan

584 Probabilistic programming languages (Stan Development Team, 2020a) - RStan package

585 Version 2.21.2 (Stan Development Team, 2020b) to implement and solve the proposed

586 Bayesian models. Specifically, the joint posterior distribution was sampled using the

587 implementation of dynamic HMC in Stan (Betancourt, 2017, 2019), as an improved version

588 of HMC algorithm (Neal, 2010; Betancourt and Girolami, 2015). In total, 4 MCMC chains

589 were used to sample each model and they were initialized randomly to ensure a better

590 exploration of the joint posterior distribution, while allowing diagnostic of the convergence.

591 Each chain consisted of 2000 samples, including a first half warm-up phase (1000 samples)

592 for the adaptation of the HMC parameters. Therefore, when combining all 4 chains, we

593 obtained 4000 samples of each parameter of the models mentioned above, drawn respectively

594 to estimate the corresponding posterior distributions. Regarding computation time, using an

595 Intel 10750H laptop CPU and parallel computation (one core per chain), dynamic HMC took

596 66s for sampling once Model\#1, 59s for Model\#2 and 53s for Model\#3 (including compiling

597 time and calculation of the diagnostics).

598 Diagnosing the HMC sampling process is a crucial step when evaluating the accuracy and

599 biases of the estimated posterior distributions. This is also known as a unique and advanced

600 feature of HMC when compared to other MCMC algorithms (Roberts and Rosenthal, 2004).

601 In this study, we considered the diagnostic approach recommended by Stan to evaluate

602 pathological behaviors of HMC sampling (Betancourt, 2017; Gabry et al., 2019; Gelman et

603 al., 2020b),

604 1) Divergent transitions for real samples drawn after the warm-up phase. This diagnostic

605 statistic is specific for the HMC sampler, mainly invigilating the miss-match between the 
606 step size of the MCMC chain and the target distribution geometries (Betancourt et al.,

607 2017). While sampling a 'high curvature' region of the target distribution, an

608 inappropriate large step size may miss-sample it, therefore biasing the resulted posterior

609 distribution. MCMC chains will approach infinite energy immediately - called divergent

610 transitions - when approaching such regions (Neal, 2010; Betancourt, 2017). These

611 divergences are recorded and reported by Stan. Note that divergence is usually related to

612 the parameterization of the model, especially when involving multivariate and

613 hierarchical structures. Parameters may usually be dependent on each other in these

614 models, therefore, creating a 'high curvature' distribution landscape, also denoted as

615 Neal's Funnel (Neal, 2003), which is difficult to sample. In order to reduce the chances

616 of such divergences, in our study, we considered reparameterization of the model into

617 non-centered forms when sampling with HMC.

618 2) The Energy-Bayesian Fraction of Missing Information (E-BFMI) is a specific 619 diagnostic statistic for HMC sampler, evaluating the efficiency of the sampling process

620 (Betancourt, 2016). Poorly chosen parameters of the HMC can decrease the efficiency of 621 the sampling process or even result in incomplete exploration of the target distribution, 622 especially when considering distributions with heavy tails. Such a behavior can be diagnosed by taking advantage of the physics feature of $\mathrm{HMC}$, by comparing the marginal energy density (denoted as $\pi_{E}$ ) and energy transition density (denoted as $\pi_{\Delta E}$ ) of the chain. When superimposing the histograms of $\pi_{E}$ and $\pi_{\Delta E}$, the higher the efficiency, the more overlap between the two distributions. The Energy Bayesian Fraction of Missing Information (E-BFMI) (Rubin, 2004) is used in Stan to quantify such comparison, by calculating the statistical expectation of the variance of $\pi_{\Delta E}$ over the variance of $\pi_{E}$. Empirically, an E-BFMI value below 0.3 is considered as problematic (Betancourt, 2016, 2017).

3) $\widehat{R}$ as a general and primary diagnostic statistic when evaluating convergence of MCMC chains (Gelman and Rubin, 1992; Brooks and Gelman, 1998). $\widehat{\boldsymbol{R}}$ is estimated for each parameter of the model as the ratio of between-chains variance over the within-

638 al., 2020).


639 Finally, we used tidyverse package (Wickham et al., 2019) in R (R Core Team, 2020) for

640 general data wrangling and visualization. Tidybayes package (Kay, 2020) was used for

641 visualizing the posterior distributions whereas bayesplot package (Gabry et al., 2019; Gabry

642 and Mahr, 2020) was used for visualizing the diagnostics of HMC chains.

\section{$643 \quad 2.7$ Statistical inferences}

644 In general, two types of statistical inferences were made in this study. To infer PAS effects on

$645 \mathrm{MEP}$ and $\mathrm{HbO} / \mathrm{HbR}$ time course, we applied the posterior predictive simulation technique in

646 which the distribution of MEP after each intervention type was estimated by feeding the fitted

647 model (e.g., Model\#1 and Model\#2) with specific pre-intervention MEP values (the same

648 approach was applied to $\mathrm{HbO}$ or $\mathrm{HbR}$ time course, respectively). Then the distribution of

649 percentage change of post-intervention MEP relative to pre-intervention MEP was used to

650 infer the PAS effects on MEP (the same approach was applied to HbO or HbR time course,

651 respectively). To infer the correlation between PAS effects on MEP and its effects on

$652 \mathrm{HbO} / \mathrm{HbR}$, we directly considered the posterior distribution of the correlation parameter in

653 Model\#3. Please find further details as follows.

654 1) When investigating the effect of PAS on MEP, we first made inferences by answering 655 the question - what will be the distribution of MEP after a certain PAS intervention when giving a specific pre-PAS MEP? This approach is more direct and convenient comparing the process checking the posterior distribution of each parameter of the model one by one. This technique is referred to as the posterior predictive simulation (Gabry et al., 2019; Gelman et al., 2020b). For instance, to infer the PAS effects on the M1 cortical excitability, we used the averaged MEP (i.e., equals to $1.0 \mathrm{mV}$, in the original data scale before normalizing) among all pre-PAS runs to represent the group-level pre-PAS M1 cortical excitability. This amplitude was then substituted into the fitted Model\#1 along with all posterior distributions of parameters (e.g., intervention-specific intercepts and slopes) to estimate a group-level post-PAS MEP distribution. By comparing the distributions of the percentage change of this post-PAS MEP distribution relative to the pre-PAS MEP amplitude, the effects of each intervention can be inferred. We also performed this inference using a set of different pre-PAS MEPs values, such as $0.2 \mathrm{mV}$, $0.6 \mathrm{mV}, 1.2 \mathrm{mV}, 2.2 \mathrm{mV}$ and $2.8 \mathrm{mV}$ according to the observed range of all individual prePAS MEP (i.e., ranging from $0.1 \mathrm{mV}$ to $3.0 \mathrm{mV}$ ), to investigate how PAS effects could be related to the pre-PAS MEP amplitude. Note that these pre-PAS MEP amplitudes were 
also scaled by dividing the global maximum value of $M E P_{o b s, s}^{\text {pre,post }}$ before being feed into the model, and posterior predicted post-PAS MEP amplitudes were rescaled back to the original data scale.

Similarly, the PAS effects on task-related hemodynamic were statistically inferred using the following steps: 1) select any preferred pre-PAS $\mathrm{HbO} / \mathrm{HbR}$ time course (e.g., the averaged $\mathrm{HbO} / \mathrm{HbR}$ of all pre-PAS runs demonstrated in the results); 2) calculate the 10 weights (variable) corresponding to this specific time courses; 3) inferring the 10 postweights along with their variance by posterior predictive simulations of the fitted hierarchical Model\#2; 4) apply a linear combination of 10 post-weight and basis functions to obtain the distribution of post-PAS $\mathrm{HbO} / \mathrm{HbR}$ time course. Note that we also

2) The correlation between M1 cortical excitability and task-related hemodynamic response can be inferred directly from the posterior distribution of the correlation parameter $\rho_{M E P-H b}$ per se. Note that this correlation distribution was estimated for each spline weight separately, therefore, the resulted posteriors can be used to infer the excitability association for each specific time point of the $\mathrm{HbO} / \mathrm{HbR}$ time course. For instance, the posterior distribution of the correlation between $\operatorname{slope} e_{[s]}^{M E P}$ and $\operatorname{slop} e_{[s]}^{H b\left(w_{5}\right)}$ indicated the relationship between the peak period (e.g., few seconds around the expected peak timing of the response) of task-related $\mathrm{HbO} / \mathrm{HbR}$ and $\mathrm{M} 1$ cortical excitability. We also conducted typical frequentist inferences of this relationship using

696 Note that for quantified statistics, we reported median and the median absolute deviation (i.e., $697 \operatorname{mad}_{s d}$ ), which was suggested by Gelman et al., 2020a and estimated as follows: $\operatorname{mad}_{s d}=$ $6981.483 \cdot \operatorname{median}_{i=1}^{n}\left|z_{i}-M\right|$, where $z_{i}$ is a certain value of a set of values $z_{i=1,2,3 \ldots n}$ and $M$ is 699 the median of all $z_{i}$. The $\operatorname{mad}_{s d}$ is a more universal representation of the variance, which is 700 comparable to the standard deviation, without considering the parametric/nonparametric 701 distribution of $z_{i}$ and is more computationally stable. 


\section{$702 \quad 2.8$ Data and code availability statements}

703 The original raw data supporting the findings of this study are available upon reasonable

704 request to the corresponding authors. fNIRS and TMS data were processed via Brainstorm

705 software (Tadel et al., 2011) available at https://neuroimage.usc.edu/brainstorm/ and the

706 fNIRS processing plugin - NIRSTORM (https://github.com/Nirstorm/nirstorm) in

707 Brainstorm. $\mathrm{R}$ code for Bayesian models is available upon reasonable request to the

708 corresponding authors.

\section{3. Results}

\section{$710 \quad 3.1$ Prior predictive simulation}

711 As illustrated in Fig.5, the resulting prior predictive simulation lines were distributed 712 symmetrically around the control line suggesting no PAS effect (i.e., intercept $=0$, slope $=1$ ).

713 This means our priors exhibited no preference towards a slope $<1$ or $>1$. Moreover, within 714 the post-PAS MEP versus pre-PAS MEP plane, the area spanned by all simulated lines 715 covered a larger area than the area enfolded by the reference lines ( 0 intercepts, a slope 716 spanning from 0.2 to 3.0). These results are confirming that priors in our hierarchical model 717 are not biased to the expected PAS effect and are more conservative than the conventional 718 MEP ratio thresholding approach. This prior predictive simulation result also applies to PAS 719 effects on $\mathrm{HbO} / \mathrm{HbR}$, since fNIRS data were normalized similarly as MEP values and the 720 priors in the multivariate linear regression models were the same.

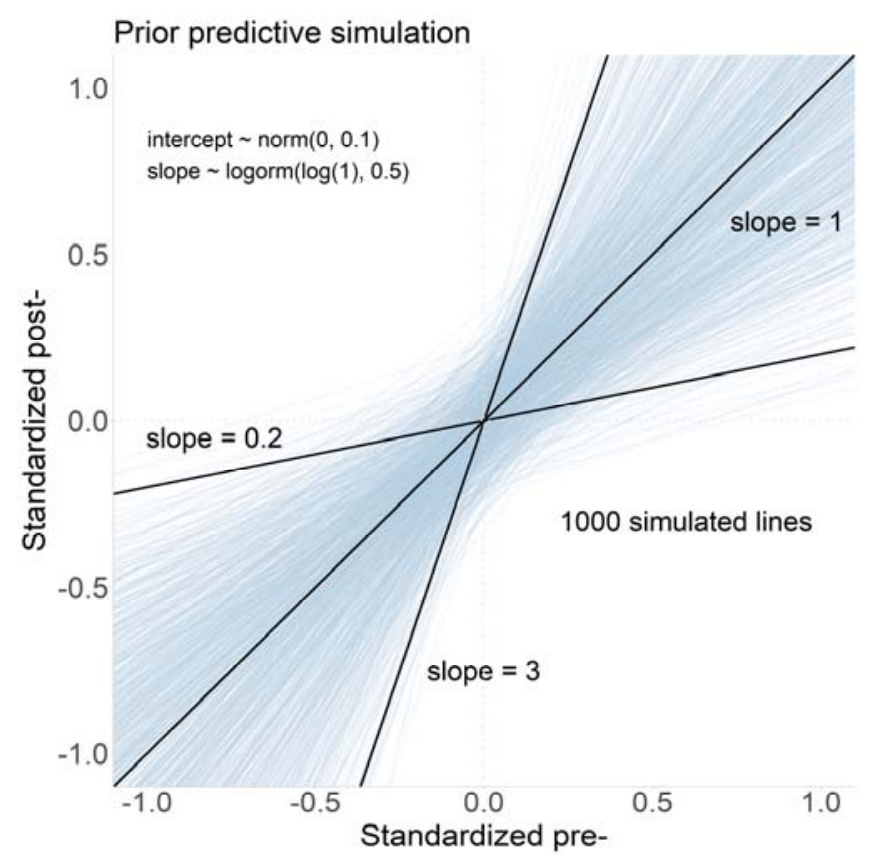


Fig.5. Prior predictive simulations for the hierarchical model of PAS effects on cortical excitability.

723 Each blue line represents one prior predictive simulation obtained by drawing simultaneously the

724 intercept and the slope parameters when considering only the priors proposed in Model\#1. For

725 comparison purposes, as a reference, we first represented a control line suggesting no PAS effect

726 (intercept of 0 , slope of 1), then two lines referring to MEP ratio outliers (intercept of 0 and slope of

7270.2 and 3 respectively.

\section{3.2 Diagnosis of HMC}

729 All of the models resulted in 0 divergences reported by Stan, indicating they were well 730 parameterized, and HMC chains explored sufficiently well the target distribution. Fig.6 731 reports the evaluation of diagnostic statistics for the two metrics $\hat{R}$ and E-BFMI. In each 732 column of Fig.6, a specific model sampling process for a specific model is being diagnosed 733 (see further details in Fig.6 caption). The first row illustrates the histogram of $\hat{R}$ for all 734 parameters in each corresponding model. No parameters resulted $\hat{R}>1.05$ indicating the 735 corresponding HMC chains indeed well converge. The second row demonstrated the 736 superimposed histograms of $\pi_{E}$ (i.e., marginal energy density) and $\pi_{\Delta E}$ (i.e., energy transition 737 density), which overlapped well for all models. This evaluation was also quantified by 738 reporting E-BFMI values for each model, which were all smaller than 0.3 . Therefore, we can 739 conclude that the HMC chains used to sample the parameters of the key models involved in 740 this study were robust and reliable according to these reported diagnostic statistics.
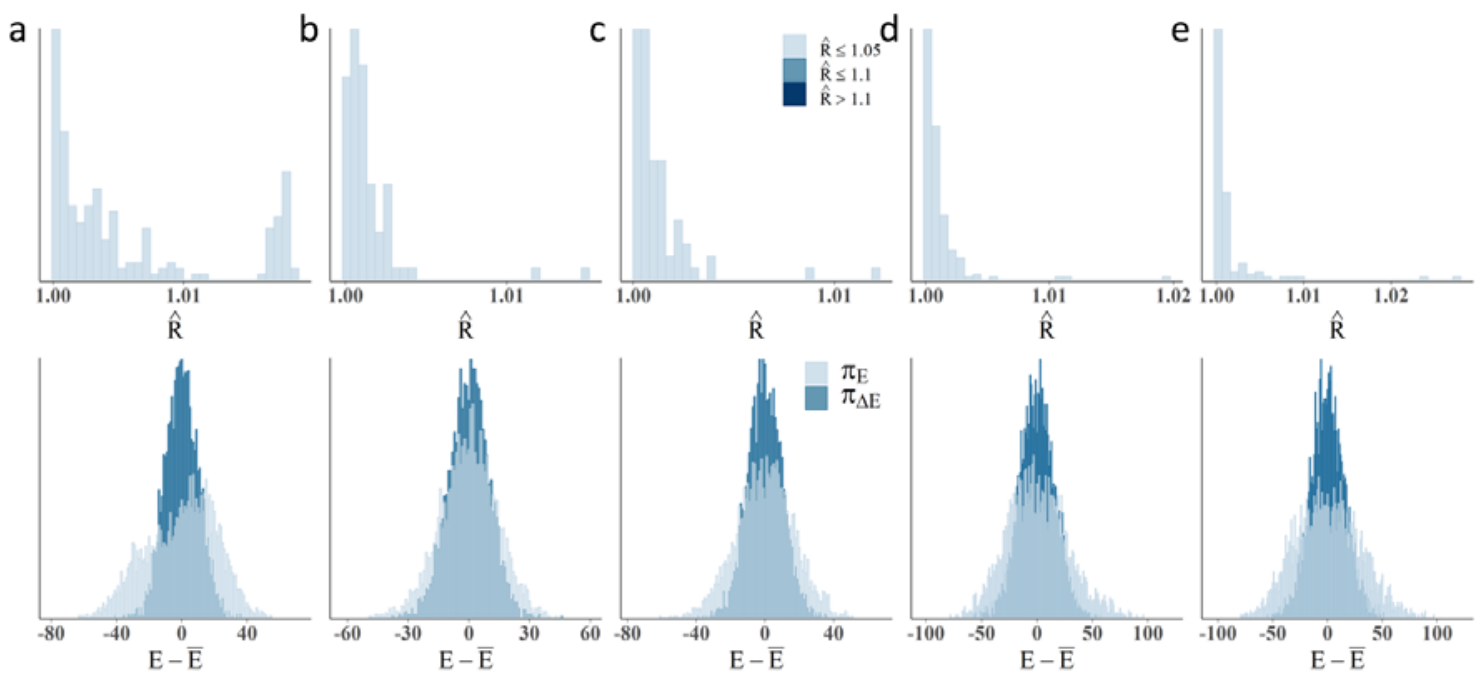

742 Fig.6. Diagnostic statistics of key features of the models considered in this study. Diagnostic statistics for a) Model\#1 - PAS effects on MEP amplitude, b) Model\#2 - PAS effects on $w_{5}$ of taskevoked $\mathrm{HbO}$, c) Model\#2 - PAS effects on $w_{5}$ of task-evoked HbR, d) Model\#3 - correlation between 
PAS effects on MEP and PAS effects on $w_{5}$ of task-evoked $\mathrm{HbO}$ and e) Model\#3 - correlation between $P A S$ effects on MEP and PAS effects on $w_{5}$ of task-evoked HbR. The first row presents the histogram of $\hat{R}$ values for all parameters among all chains of each model. No $\hat{R}$ value was above 1.05, suggesting that all chains converged well. The second row presents the superimposed distributions of the marginal energy density $\pi_{E}$ and the energy transition density $\pi_{\Delta E}$ for all HMC chains sampled for each model. The corresponding quantification metric E-BFMI was smaller than 0.3, indicating a good overlapping between the two distributions.

\subsection{PAS effects on cortical excitability}

When considering Model\#1, the estimated regression lines (using the averaged intercept and slope parameters calculated from their posterior distributions) linking pre- and post-PAS MEPs for each intervention are reported in Fig.7a. The regression line estimated for sham intervention (black line) was found as expected - between the regression lines estimated for PAS25 (red line) and PAS10 (blue line), and it was almost identical to the reference line reporting no effect (intercept=0, slope=1). Observed pairs of post-PAS MEP and pre-PAS MEP mean amplitude over all trials are presented as solid pints (observed data), whereas corresponding estimated 'true' amplitudes are presented as empty points. The black lines connecting each pair of solid (observed mean) and empty (estimated 'true' mean) points illustrate the shrinkage process, also known as the result of partial pooling obtained when considering hierarchical Bayesian modeling. This demonstrated the regularization property of the model, where the estimated 'true' MEPs corresponding to each intervention group shrank toward the corresponding regression line. Moreover, when considering the variance of the MEPs, the larger the MEP variability of a certain run, the more shrinkage there was. Results of posterior predictive simulation at the group-level, when considering a pre-PAS MEP amplitude of $1.0 \mathrm{mV}$ is illustrated in Fig. $7 \mathrm{~b}$, as the posterior distribution of relative changes of post-PAS MEP amplitudes (in \%) after each intervention. PAS25 intervention resulted in a substantial relative increase of post-PAS MEP amplitude (median $\pm \operatorname{mad}_{s d}=30.6 \% \pm$ $14.6 \%)$, consisting of a posterior probability of 0.97 for obtaining an increase in MEP amplitude. The posterior distribution of sham post-PAS MEP amplitude exhibits a nice symmetric pattern around a $0 \%$ increase (an increase of $2.3 \% \pm 14.5 \%$ ). The effects of PAS10 were subtle, showing a slight shift towards the negative side consisting in a relative decrease of $-1.80 \% \pm 11.0 \%$, and a probability of 0.57 of obtaining a decrease in MEP amplitude. Individual-level inferences are presented in Fig.S1, where both PAS25 and PAS10 effects are showing a large between-subject variability, as addressed in the introduction. 
778 When reporting individual level results, posterior predictive simulations were obtained by considering the same averaged pre-PAS MEP amplitude over 40 sessions as the input for all

780 subjects, taking full benefit of hierarchical modeling, allowing to compare individual-level 781 results within the appropriate framework.
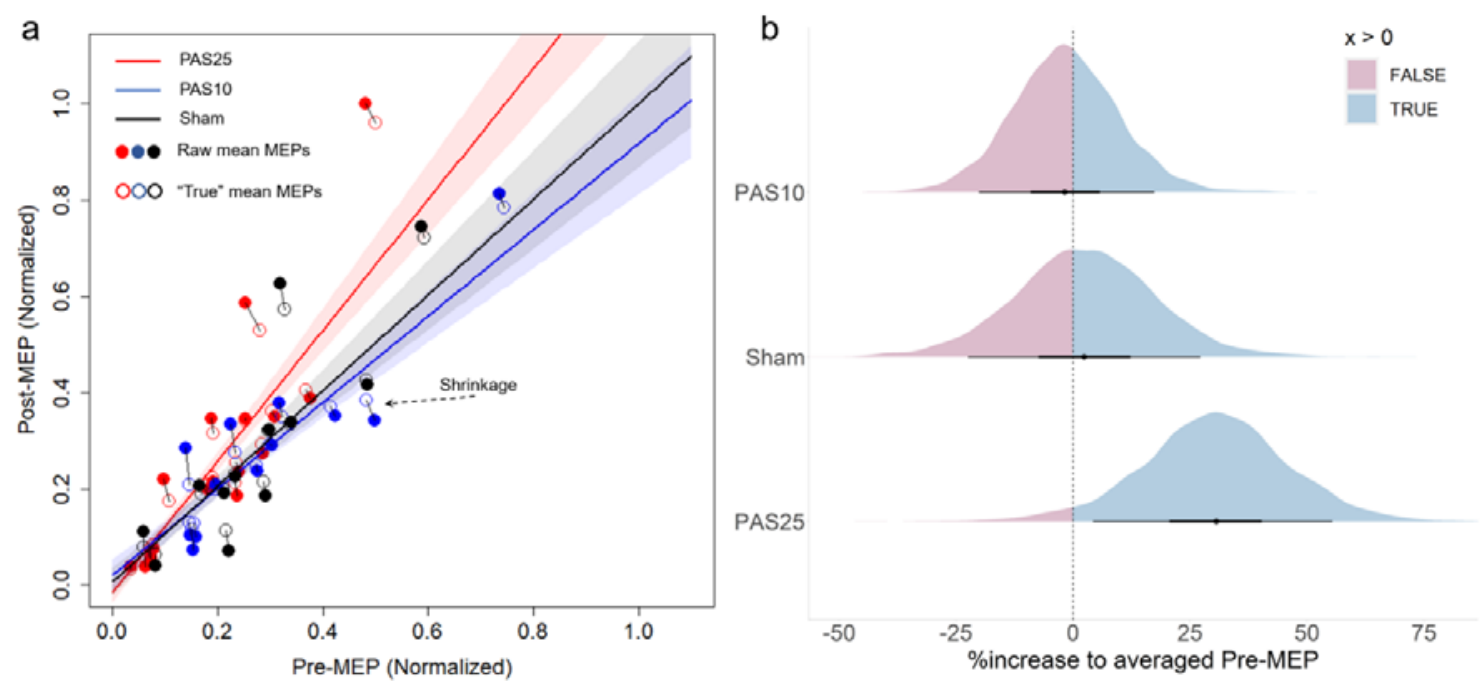

783 Fig.7. PAS effects on cortical excitability. a) the regression lines of each intervention estimated by 784 the mean of intercept and slope from the corresponding posterior distribution, PAS25 (red), PAS10 785 (blue) and sham (black). Pre-and post-PAS MEP amplitudes were normalized by dividing by the 786 global maximum amplitude of all 80 MEP values. Shadow areas represent the 50\% interval estimated 787 from the posterior distribution of the regression parameters. Solid points correspond to pairs of 788 averaged pre-/post-PAS MEP amplitudes over all trials of each specific run. Empty points represent 789 the 'true' amplitude of the corresponding pre-/post-PAS MEP pair estimated using the proposed 790 hierarchical Bayesian Model\#1. The black bar connecting each solid point to the corresponding 791 empty point illustrates the shrinkage process of Bayesian inference of the hierarchical model; $b$ ) 792 Posterior predictive simulations of post-PAS MEP amplitudes obtained when considering a given pre-

793 PAS MEP amplitude of $1 \mathrm{mV}$ as input, corresponding to the averaged pre-PAS MEP amplitude over 794 all 40 sessions. The blue area represents the probability of obtaining a relative increase (in \%) for the 795 post-PAS MEP amplitude when compared to the pre-PAS MEP amplitude, whereas the pink area 796 represents the probability of obtaining a relative decrease (in \%). The black dot represents the 797 median of each posterior distribution, and the surrounding bars show the corresponding 50\% and $79890 \%$ credibility intervals. 

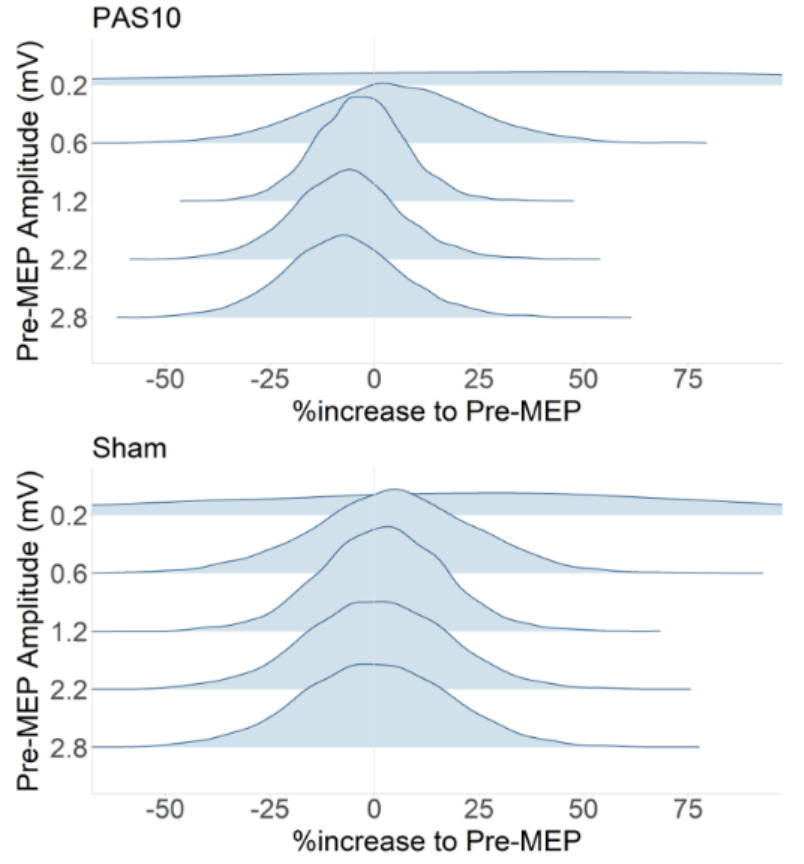

PAS25

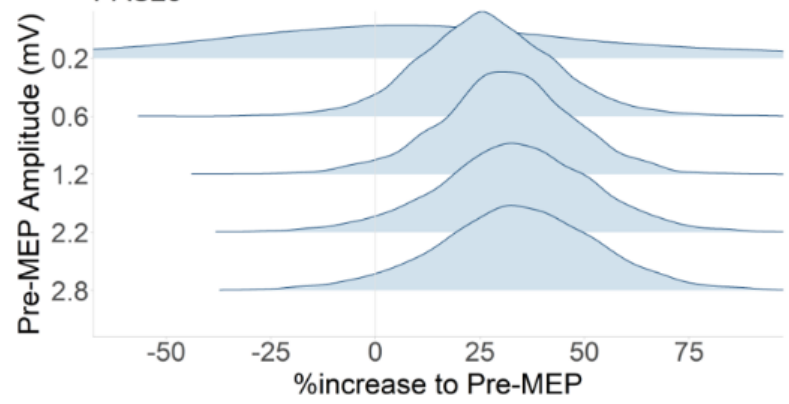

800 Fig.8. Effects of spTMS intensity on PAS assessment. We used posterior predicting simulations

801 applied to five levels of pre-PAS MEP amplitudes, to evaluate the impact of five levels spTMS

802 intensities. Posterior distributions of the corresponding relative changes in post-PAS MEP amplitude

803 relative to pre-PAS MEP amplitudes are presented in each row. The expected effects of PAS 25

804 (positive \% increase) and PAS10 (negative \% decrease) became clearer when increasing the spTMS

805 intensity. On the other hand, when considering the sham intervention, we found no effect of relative

806 changes in post-PAS MEP amplitude, exhibited at all intensity levels, as well as symmetric

807 distributions around 0\%.

808 Fig.8 presents the effects of simulating different pre-PAS MEP amplitudes as inputs, on the 809 relative change of post-PAS MEP amplitude for each intervention, at the group level. For 810 both PAS25 and PAS10, the higher the pre-PAS MEP amplitude was, the higher the relative 811 change in MEP amplitude was. In further details, PAS25 resulted in an increase of post-PAS 812 MEP amplitude of 813

),

( ) when considering 
814 an input pre-PAS MEP amplitude of $0.6 \mathrm{mV}, 1.2 \mathrm{mV}, 2.2 \mathrm{mV}$ and $2.8 \mathrm{mV}$, respectively.

815 Similarly, PAS10 resulted in an increase of post-PAS MEP amplitude of $+4.2 \% \pm 18.9 \%$

816 (Prob $=0.59)$, when considering an input pre-PAS MEP amplitude of $0.6 \mathrm{mV}$, followed

817 respectively by decreases of $-3.1 \% \pm 10.5 \%(\operatorname{Prob}=0.62),-6.5 \% \pm 12.6 \%(\operatorname{Prob}=$

$8180.70)$ and $-7.51 \% \pm 13.8 \%($ Prob $=0.71)$ when considering an input pre-PAS MEP

819 amplitude of $1.2 \mathrm{mV}, 2.2 \mathrm{mV}$ and $2.8 \mathrm{mV}$. This important finding of our proposed Bayesian

820 Model\#1 suggests that even without increasing the TMS stimulation intensity during PAS,

821 simply increasing the spTMS intensity considered to measure changes in excitability can

822 reveal the expected PAS effects more clearly, while reducing some variability in the data. On

823 the other hand, when assessing this effect on sham, we obtained similar distributions of

824 relative changes in post-PAS MEP amplitude, all symmetric around $0 \%$, consisting in relative

825 changes of $4.6 \%, 2.0 \%, 0.6 \%$ and $0.2 \%$, when considering a pre-PAS MEP amplitude of

$8260.6 \mathrm{mV}, 1.2 \mathrm{mV}, 2.2 \mathrm{mV}$ and $2.8 \mathrm{mV}$, respectively. Importantly, the higher the pre-PAS MEP

827 amplitude was, the closer to $0 \%$ the median of relative change in post-PAS MEP amplitude

828 was. Overall, when considering pre-PAS MEP amplitude of $0.2 \mathrm{mV}$ for each intervention, we

829 found a large level of uncertainty in spTMS responses, suggesting that small MEP amplitude

830 induced by spTMS should be avoided when assessing the level of brain excitability.

\section{$831 \quad 3.4 \mathrm{PAS}$ effects on task-related $\mathrm{HbO} / \mathrm{HbR}$ responses}

832 Fig.9 showed the PAS effects on the whole time course $\mathrm{HbO} / \mathrm{HbR}$, as a contrast (i.e., 833 subtraction of posterior distributions) between the intervention of interest (PAS25 or PAS10) 834 and sham condition. When considering the group level averaged pre-PAS $\mathrm{HbO} / \mathrm{HbR}$ 835 responses (normalized to $[-1,1]$ ) as input for posterior predictive simulations (dashed red and 836 blue curves for $\mathrm{HbO}$ and $\mathrm{HbR}$ ), we observed that PAS25 (Fig.9a) resulted in a relative 837 increase of $\mathrm{HbO}$ amplitude (solid red curve) and HbR amplitude (solid blue curve), mainly 838 around the expected peak of the hemodynamic response (from 8s to 16s). When comparing 839 absolute peak amplitudes, the probability of increasing the hemodynamic response after 840 PAS25 was 0.80 for $\mathrm{HbO}$ response and 0.82 for HbR response. After PAS10 (Fig.9b), our 841 results are suggesting at the group level a subtle relative decrease of $\mathrm{HbO}$ and $\mathrm{HbR}$ absolute 842 amplitudes around the peak of the hemodynamic response. The probability of obtaining a 843 relative decrease in absolute peak amplitudes after PAS10 was 0.66 for $\mathrm{HbO}$ response and 8440.48 for HbR response. Interestingly, PAS10 demonstrated a clear absolute amplitude 845 decrease within a period ranging from the peak to the end of the response (11s to $25 \mathrm{~s}$ ) for 846 both $\mathrm{HbO}$ and $\mathrm{HbR}$. 

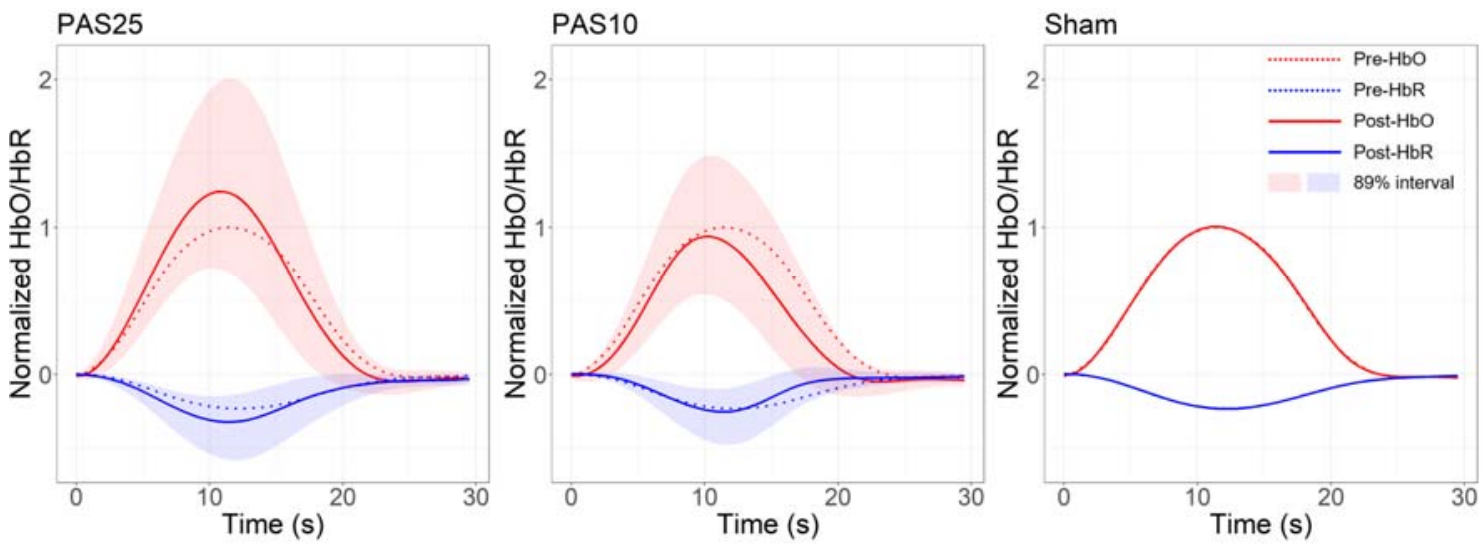

848 Fig.9. PAS effects on the whole time course of HbO/HbR. Posterior predictive simulations of post-

849 PAS HbO/HbR time course (solid curves: $\mathrm{HbO}$ in red and HbR in blue) when considering pre-PAS

$850 \mathrm{HbO/HbR}$ template input defined as the group-level averaged pre-PAS HbO/HbR response

851 (normalized to $[-1,1]$ ) over all 40 sessions (dash curves). The shadow area represents the 89\%

852 credibility interval of resulted post-PAS HbO/HbR responses. Note that sham effects were subtracted

853 from the PAS25 and PAS10 to obtain so-called 'unbiased' effects. The overlappings of lines in the

854 sham panel are shown as a sanity check of the contrast.

855 3.5 Relationship between PAS effects on task-related cortical hemodynamic 856 activity and PAS effects on M1 excitability

857 Fig.10a presents inferences on the relationship between PAS effects on task-related cortical

858 hemodynamic activity and PAS effects on M1 excitability, represented by the posterior

859 distribution of correlations between the slope of MEP amplitudes (post-PAS versus pre-PAS)

860 and the slope of spline weight $w_{5}$ (post-PAS versus pre-PAS) for either $\mathrm{HbO}$ or $\mathrm{HbR}$ task-

861 related responses. Since our previous observations of the PAS effects were conducted for the

862 whole $\mathrm{HbO} / \mathrm{HbR}$ time course (Fig.9), we selected $w_{5}$ as the spline weight of interest

863 considering it corresponded to the spline basis function exhibiting a peak at $12.5 \mathrm{~s}$, therefore

864 consisting in the closest temporal pattern when compared to the expected hemodynamic

865 response. The probability of obtaining a positive correlation between PAS effects on MEP

866 amplitude and PAS effects on $\mathrm{HbO}$ response was 0.77 ; and 0.79 for a positive correlation

867 between PAS effects on MEP amplitude and PAS effects on HbR response. The

868 corresponding $89 \%$ highest posterior density interval (HPDI) of this correlation was [-0.26,

869 0.89] for $\mathrm{HbO}$; and [-0.20, 0.84] for HbR. Fig.10b presents the linear fits between the

870 averaged slope $e_{[s]}^{M E P}$ and the averaged slope $e_{[s]}^{H b\left(w_{5}\right)}$ obtained for each session $s$ among all 40 
871 sessions. The corresponding estimated Pearson's correlation was 0.58 for MEP vs. HbO (p<

$\left.872.0001, \mathrm{CI}_{95 \%}=[0.33,0.75]\right)$ and 0.56 for MEP vs. $\mathrm{HbR}\left(\mathrm{p}<.001, \mathrm{CI}_{95 \%}=[0.30,0.74]\right)$.

a
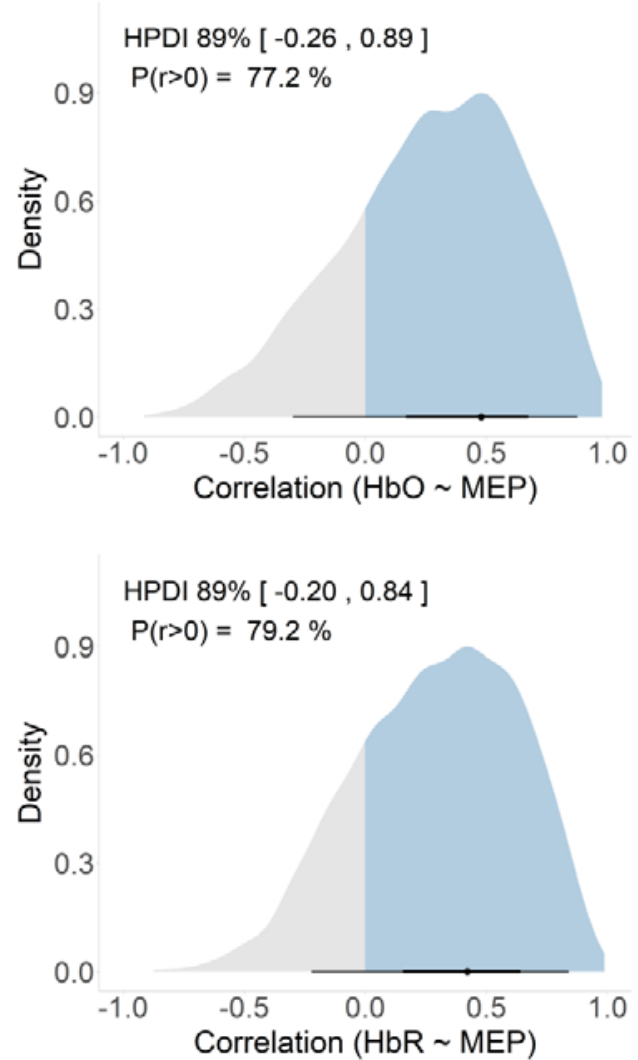
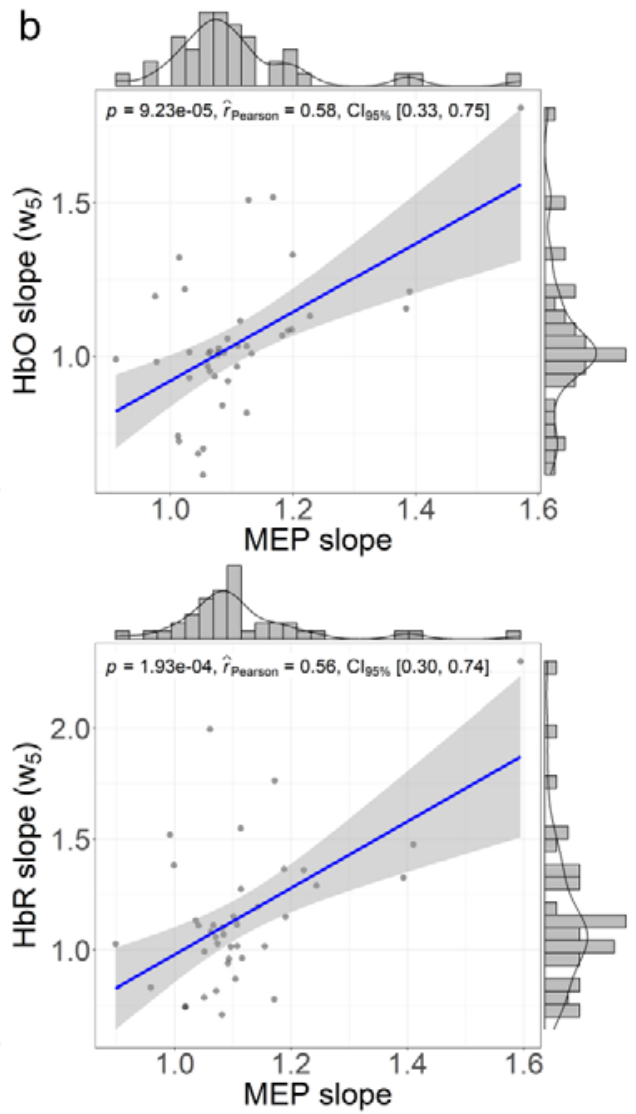

874 Fig.10. The relationship between task-related cortical hemodynamic activity and M1 excitability. a)
875 the posterior distribution of the correlation between slope $e_{[s]}^{M E P}$ and $\operatorname{slop} e_{[s]}^{H b\left(w_{5}\right)}$ for $H b O$ (top) and

$876 \mathrm{HbR}$ (bottom). The blue area represents the probability of observing a positive correlation $(\rho>0)$. The

877 black dot represents the median of each posterior distribution, and the surrounding bars show the

878 corresponding $50 \%$ and $90 \%$ credibility intervals. b) Linear fit (blue line) between the averaged 879 slope $_{[s]}^{M E P}$ and the averaged slope ${ }_{[s]}^{H b\left(w_{5}\right)}$ obtained for all 40 sessions (each represented by a grey 880 dot). The grey area indicates the 95\% confidence interval of the regression. Estimated Pearson's 881 correlation betweenslope $e_{[s]}^{M E P}$ and slope $e_{[s]}^{H b\left(w_{5}\right)}$ over the 40 sessions together with corresponding $p$ 882 values and 95\% confident intervals are shown on top of each panel. The marginal histograms and 883 fitted density functions are shown on the side of each corresponding marginal axis.

884 Fig.11 illustrates the posterior distribution of the correlation between $s l o p e_{[s]}^{M E P}$ and $885 \operatorname{slope}_{[s]}^{H b\left(w_{n}\right)}$, when considering each spline weight for $n=2,3,4,5,6,7$ and 8 . The closer the corresponding peak of the spline basis function associated with the weight $w_{n}$ was to the 

median value of $0.06,0.17,0.31,0.32,0.15,-0.06$ and 0.03 when considering for $\mathrm{HbR}$, therefore confirming this trend. Our results are suggesting that the expected positive correlation between PAS effects on task-related hemodynamic response and PAS effects on

893 M1 excitability appeared mostly around the peak of $\mathrm{HbO} / \mathrm{HbR}$ time course (e.g., ), in 894 agreement with PAS effects reported previously in Fig.9. On the other hand, for the earliest aspects of the hemodynamic response (modeled using ) as well as for the end of the response (modeled using ), we found a posterior correlation with a median close to zero, suggesting no relationship between and for the corresponding time 898 periods.
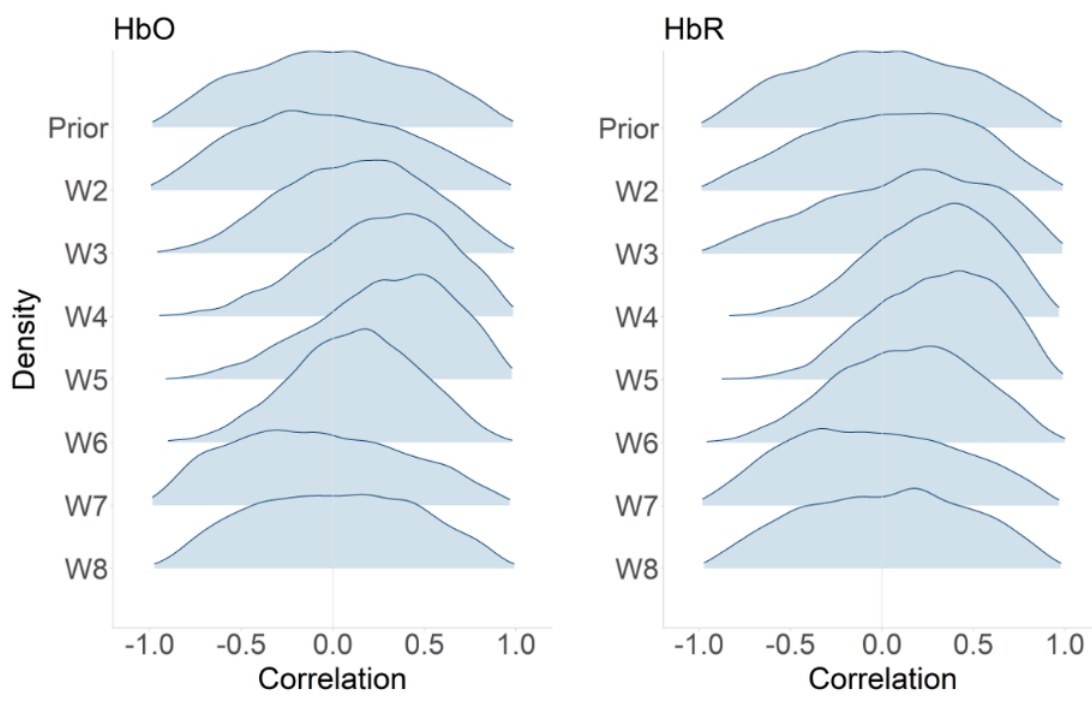

900 Fig.11. Posterior distributions of the correlations between

and several selected

903 The prior distribution of the correlation (i.e., LKJ(2)) on the first row demonstrates a perfect 904 symmetric to the 0 correlation. There was a trend showing the closer the corresponding peak of the 905 spline basis function associated with the weight was to the expected peak of the HbO/HbR 906 response, the higher the correlation between and showed the highest 907 correlation values for both $\mathrm{HbO}$ and $\mathrm{HbR}$ as it corresponded to the spline basis function exhibiting 908 the peak at 12.5s, therefore the closest temporal pattern when compared to the expected 
909 hemodynamic response. In contrast, the earliest aspects of the hemodynamic response (modeled

910 using $w_{2,3}$ ) and the end of the response (modeled using $w_{7,8}$ ), showed almost identical distributions

911 when comparing to the prior.

\section{4. Discussion}

\section{$913 \quad 4.1$ PAS effects on cortical excitability}

914 Using hierarchical Bayesian modeling, we first investigated PAS effects on cortical

915 excitability, which was measured using MEP amplitude induced by spTMS. Probability

916 distributions of the relative changes (in \%) of post-PAS MEP amplitudes, when compared to

917 pre-PAS MEP amplitudes, were estimated using posterior predictive simulations. Our results

918 showed a substantial increase of MEP amplitude after PAS25, a subtle decrease after PAS10

919 and no changes after control (sham). These results are consistent with previous PAS studies

920 (Stefan, 2000; Wolters et al., 2005; Tsang et al., 2015; Lee et al., 2017; Suppa et al., 2017).

921 These studies considered conventional MEP analysis, calculating the ratio between the

922 averaged MEP amplitude after PAS over the one before PAS. Therefore, when MEP ratio

923 was larger than 1 , it indicated an excitability increase and vice versa. In contrast, here we

924 applied a full Bayesian workflow using an advanced sampling algorithm. The benefits of this

925 procedure are: 1) multivariate linear regression allowed the differentiation of interventions

926 and subjects, hence modeling the heterogeneity of intervention effects exhibited in different

927 groups of data; 2) involving intercept in linear regression reduced the influences of low MEP

928 amplitudes runs when compared to the conventional ratio calculation of post- over pre-PAS

929 MEPs; 3) the variability of MEP amplitudes were considered in the estimation of the PAS

930 effects rather than only using the averaged amplitudes of each run and ignore the variance; 4)

931 parameters of the model were estimated by Bayesian inferences using dynamic HMC

932 algorithm sampling posterior distributions using a hierarchical structure and weakly informed

933 priors, therefore, allowing partial pooling to reduce the estimation uncertainty; 5) flexible and

934 intuitive statistical inferences of the modeled PAS effects were obtained by conducting

935 posterior predictive simulations from the model learned from the data. This means by giving

936 any pre-PAS MEP amplitude and intervention index, the distribution of the corresponding

937 group-level post-PAS MEP amplitude could be estimated; Finally, 6) the estimated PAS

938 effects were reliable and informative, as suggested by their posterior probability distributions,

939 rather than considering only a statistical significance test providing a dichotomous output. 
940 Moreover, our model also allowed inferring the effects of MEP amplitude itself on the effect

941 size of the resulted excitability changes modulated by PAS. In Fig.8, we reported a pattern

942 suggesting that the higher the MEP amplitude was, the larger was the effect size of both

943 PAS25 and PAS10. This pattern was not biased when comparing to the sham session, which

944 showed no effects for different pre-PAS MEP amplitudes. It is important to mention that

945 when considering posterior predictive simulation, the intensity of the TMS pulse during the

946 intervention session (PAS25 or PAS10 or sham) did not change. This means the underlying

947 intervention effects did not change. Then considering spTMS as the assessment procedure to

948 measure brain excitability, our results are suggesting that a high enough spTMS intensity

949 might help to measure more accurately PAS effects. Please also note that the pre-PAS MEP

950 amplitudes $(0.2 \mathrm{mV}, 0.6 \mathrm{mV}, 1.2 \mathrm{mV}, 2.2 \mathrm{mV}$ and $2.8 \mathrm{mV})$ used in this posterior predictive

951 simulation were selected based on the observed range $(0.1 \mathrm{mV}$ to $3.0 \mathrm{mV})$ of all individual

952 pre-PAS MEP. This observed range of MEP from our data is also consistent with the spTMS

953 evoked MEP distribution estimated by a recent meta-analysis study in which 687 health

954 subjects' data were considered among 35 studies (Corp et al., 2021). More importantly, this

955 meta-analysis study also investigated the relationship between the baseline MEP (referred to

956 as the test stimulation) amplitude and the short interval intracortical inhibition (SICI) by

957 polling data from 15 studies consisted of 295 healthy subjects. They showed a significant

958 negative relationship between the baseline MEP amplitude and SICI, suggesting that "SICI is

959 best probed by high relative test stimulation intensities" (Corp et al., 2021). This result is also

960 concordant with our finding on PAS10, in which the higher the pre-PAS MEP (test

961 stimulation) is, the more PAS10's inhibitory effects (decreased post-PAS MEP comparing to

962 the pre-PAS MEP) are pronounced. This consistency demonstrated the power of our

963 Bayesian analysis to infer similar findings from relatively small sample-sized data when

964 comparing with meta-analysis results consisting of a much larger sample size.

\section{4.2 PAS effects on the whole HbO/HbR time course of finger tapping responses}

966 To our best knowledge, our study demonstrated for the first time PAS effects on the whole

967 time course of task-related $\mathrm{HbO} / \mathrm{HbR}$ time courses. In contrast, few time segments along 968 selected time windows were considered in previous studies, then $\mathrm{HbO}$ or $\mathrm{HbR}$ amplitudes

969 were just averaged within each time segment and compared before and after interventions

970 (Chiang et al., 2007; Yamanaka et al., 2010). In our previous study (Cai et al., 2021b) we also

971 simply averaged the $\mathrm{HbO} / \mathrm{HbR}$ amplitude within a 5s long time window centered around the

972 peak of the hemodynamic response to represent the total amount of hemoglobin delivered to 
973 the region of interest. The Bayesian approach proposed in this study brings more insight into

974 the investigation of PAS effects on hemodynamics, considering not only the peak amplitude

975 before and after interventions, but whole $\mathrm{HbO} / \mathrm{HbR}$ time courses. For instance, visual

976 inspections of results presented in Fig.9 are suggesting that PAS effects are indeed more

977 pronounced around the peak of the expected hemodynamic response. This is expected if we

978 can assume that the hemodynamic response function (HRF) is not much affected by

979 interventions, then the expected task-related hemodynamic response would result from a

980 convolution with a higher or lower amplitude boxcar function representing excited or

981 inhibited neuronal activity patterns (Sotero and Trujillo-Barreto, 2007). Therefore, the effect

982 of intervention should appear mostly around the peak, and the closer to the peak the higher

983 the effect size. Consequently, averaging $\mathrm{HbO}$ or $\mathrm{HbR}$ amplitude within a certain time

984 window would 'dilute' the estimation of the effect of interest, especially when considering

985 the effect size was not large, for instance around 25\% increase for HbO after PAS25 is shown

986 in Fig.9.

987 The fact that PAS intervention effects could be observed mainly around the peak of

988 hemodynamic time courses may also explain the difficulty of investigating similar questions

989 using fMRI. Indeed, a typical BOLD signal is sampled around $0.5 \mathrm{~Hz}$ using standard fMRI

990 sequences. Such low temporal resolution may not be sufficient to sample well the effects

991 around the peak and could possibly explain why no PAS effects were found on BOLD signal

992 changes in the PAS and fMRI study reported by Kriváneková et al., 2013. Besides, depending

993 on how well fMRI BOLD samples and the actual peak of the hemodynamic response are

994 phased-locked, the mismatch between the time of BOLD signal sampling and the actual peak

995 of the response may introduce some confounds, when comparing BOLD signal changes

996 before and after PAS interventions. Another benefit of modeling accurately the whole

$997 \mathrm{HbO} / \mathrm{HbR}$ time course is the possibility to offer alternative interpretations of PAS effects. For

998 instance, our results in Fig.9 showed a slight time shift for $\mathrm{HbO}$ after PAS25 and a larger one

999 after PAS10 (e.g., the peak time of HbO shifted from 12s to 10s after PAS10). The decrease

1000 of $\mathrm{HbO}$ amplitudes after PAS10 was also mainly exhibited from 11 s to $25 \mathrm{~s}$ of the response

1001 time courses. These observations may suggest a more complex mechanism of the effect of

1002 neuronal plasticity on neurovascular coupling. Further analysis using the deconvolution

1003 technique (Machado et al., 2021) to estimate HRFs that are related to these hemodynamic

1004 responses may help us to better investigate such a potential mechanism but it is beyond the

1005 scope of this study. 
1006 It is important to mention that we did not perform a specific analysis for every time sample of

1007 the hemodynamic response. We regularized and reduced the dimensionality of the problem

1008 by projecting $\mathrm{HbO} / \mathrm{HbR}$ responses on $\mathrm{B}$-splines as temporal basis functions. Therefore, PAS

1009 effects on hemodynamic were modeled by only 10 weights instead of 60 data points, whereas

1010 the actual post-PAS $\mathrm{HbO} / \mathrm{HbR}$ time courses could then be fully retrieved from the estimated

1011 weights and the spline basis functions. The choice of the number and locations of knots might

1012 have limited the 'resolution' of our proposed correlation analysis. There are more advanced

1013 Bayesian spline approaches, such as the penalized spline (P-spline) (Eilers and Marx, 2010;

1014 Ventrucci and Rue, 2016), which introduces an extra prior to regularize the number of

1015 effective knots. This approach was mainly designed to smooth a time course and prevent

1016 overfitting rather than considering an accurate representation of the time course. Non-

1017 parametric time series modeling techniques were also proposed in this context, without

1018 assuming the location of the knots along the time course. For instance, Gaussian process

1019 regression (Neal, 1998) characterizes the time course, such as the hemodynamic response, as

1020 an unknown function. Samples of the time course are then drawn from a multinormal

1021 distribution providing a full covariance matrix of all time samples. Our analysis could benefit

1022 from these non-parametric approaches to avoid eventual limitations associated with the

1023 choice of the knots, but this was beyond the scope of this study.

1024 It is also worth noting that these results of PAS effects on the whole $\mathrm{HbO} / \mathrm{HbR}$ time courses

1025 were also benefiting from accurate time courses estimated by our previously proposed fNIRS

1026 reconstruction workflow (Cai et al., 2021c). In this workflow, the fNIRS acquisition montage

1027 was personalized and the detection sensitivity of it was maximized to the individual ROI.

1028 Meanwhile, the MEM framework adapted from our previous works in the context of electro-

1029 /magneto-encephalogram source imaging (Chowdhury et al., 2013, 2016; Grova et al., 2016;

1030 Heers et al., 2016; Hedrich et al., 2017; Pellegrino et al., 2020) for conducting fNIRS

1031 reconstruction (Cai et al., 2021a) also ensured accurate estimation of $\mathrm{HbO} / \mathrm{HbR}$ time courses

1032 from reconstructed spatiotemporal maps. For instance, delays between $\mathrm{HbO}$ and $\mathrm{HbR}$ peak

1033 times were around 1s shown in Fig.9, which is consistent with our previous finding (Cai et

1034 al., 2021c) and fNIRS literature (Jasdzewski et al., 2003; Steinbrink et al., 2006).

\section{4.3 Relationship between PAS effects on task-related hemodynamic and PAS 1036 effects on M1 excitability}

1037 We also investigated the relationship between PAS effects on task-related hemodynamic 1038 activity and PAS effects on cortical excitability along the whole $\mathrm{HbO} / \mathrm{HbR}$ time course. 
1039 When compared to standard frequentist analysis of the correlation between slope $\mathrm{MEP}_{[s]}$ and

$1040 \operatorname{slope}_{[s]}^{H b\left(w_{n}\right)}$ reported in Fig.10b, our proposed Bayesian analysis was more informative since

1041 we could estimate the whole posterior distribution of such a correlation, instead of providing

1042 a single correlation value estimated from the mean effects while ignoring the variance. Even

1043 if the sample size was not large and we acknowledge the influences from large variability of

1044 PAS effects and variability of task-evoked hemodynamic responses, our proposed

1045 hierarchical Bayesian models were able to demonstrate a high probability of positive

1046 correlations between MEP and hemodynamic slopes (representing the PAS effects), around

1047 the peak of $\mathrm{HbO}$ and $\mathrm{HbR}$ responses. This finding is consistent with previous results reported

1048 in animal studies, suggesting a positive correlation between hemodynamic responses and

1049 cortical excitability. For instance, Allen et al., 2007 demonstrated decreased oxygenations in

1050 anesthetized cat's visual cortex after applying inhibitory rTMS; increased fMRI resting-state

1051 connectivity, GABA, glutamine and glutamate levels after performing excitatory rTMS.

1052 Reduced connectivity and glutamine levels after applying inhibitory rTMS on healthy rat's

1053 right hemisphere were reported by Seewoo et al., 2019. The reliability of our proposed model

1054 was further confirmed by the fact that no correlation was found between PAS effects on MEP

1055 and hemodynamic responses, when considering other time windows, such as the initial

1056 aspects and the end of the hemodynamic response. The further the analyzed time period was

1057 to the expected hemodynamic peak, the closer to the prior was to the posterior distribution of

1058 the correlation, with no preference on either positive or negative correlations.

\section{$1059 \quad$ 4.4 HMC sampling and diagnostic}

1060 Taking advantage of dynamic HMC to sample the hierarchical Bayesian models in this study, 1061 we were able to carefully diagnose the pathological behavior of MCMC sampling chains 1062 (Betancourt, 2017, 2019). This diagnostic procedure is an essential step when applying 1063 Bayesian data analysis (Gelman et al., 2020b). To allow accurate and reliable inferences, 1064 MCMC chains must explore well the typical set of the posterior distributions, in which most 1065 of the probability density is contained. For instance, the convergence of MCMC chains needs

1066 to be confirmed and quantified to ensure such full explorations. When inappropriate 1067 parameters of the chain are chosen (e.g., the step size), abnormalities such as divergences 1068 should be detected to avoid eventual sampling biases. In our study, we reported several 1069 diagnostic statistics for all key components of three models using both visualization and 1070 quantified metrics, following the recommendations of the Stan team (Gelman et al., 2013; 
1071 Gabry et al., 2019; Stan Development Team, 2020a). The proposed diagnostic statistics

1072 considered here also constitute a unique feature of HMC sampling, when compared to

1073 conventional MCMC algorithms such as Gibbs sampling (Geman and Geman, 1984; Gelfand

1074 and Smith, 1990). HMC is also considered to be more accurate by taking advantage of

1075 sampling all parameters at the same time, comparing to Gibbs that often samples parameters

1076 alternatively one after the other which may bias the resulted posterior distribution due to the

1077 inherent correlations between parameters. Overall, the diagnostic analysis of the sampling

1078 process in this study is suggesting that our inferences are built upon well-sampled posterior

1079 distributions, reasonably accurate and unbiased. Similar HMC sampling and diagnostic

1080 approaches were also reported in several recent studies, such as a Bayesian virtual epileptic

1081 patient to model the spread of epileptic activity (Hashemi et al., 2020); a Bayesian latent

1082 spatial model for mapping biomarkers of the progression of Alzheimer's disease (Dai et al.,

1083 2021); the Bayesian multilevel modeling to improve statistical inferences in fMRI analysis

1084 (Chen et al., 2019b, 2019a, 2021) and a hierarchical Bayesian model to investigate

1085 mechanisms of reinforcement learning and decision-making (Ahn et al., 2017).

\section{$1086 \quad 4.5$ Limitations and perspectives}

1087 While the Bayesian approach is known to improve the uncertainty of statistical inferences 1088 when dealing with small samples data set, as illustrated by our results, there is no doubt that 1089 this study would benefit from a larger sampling size. Conventional frequentist power 1090 analyses (Bhalerao and Kadam, 2010) could estimate the minimum number of samples 1091 required to obtain a significant effect for a statistical power such as $80 \%$. When considering 1092 the standard deviation of MEP amplitudes and fMRI BOLD signals reported in PAS (López1093 Alonso et al., 2014) and in fMRI literature (Kriváneková et al., 2013), we estimated that 1094 when assuming random sampling, at least 50 subjects would be required for MEP analysis 1095 and more than 100 subjects would be required for hemodynamic analysis using fMRI BOLD.

1096 Despite the inherent limitation of power analysis which may underestimate the sample size 1097 (Gelman and Carlin, 2014), in practice, it is challenging to conduct acquisitions with this 1098 amount of subjects especially considering all three different interventions.

1099 We only involved one model for each investigation in this study. It is indeed recommended to 1100 construct multiple models based on different hypotheses of the same question and then 1101 quantitatively compare these models using techniques such as cross-validation to choose the 1102 most reliable one, providing a trade-off between overfitting and underfitting (Gelman et al., 1103 2020b). For instance, we proposed a linear relationship between cortical excitability and 
1104 hemodynamic responses evoked by a finger-tapping task. However, such association might 1105 reach a plateau when excitability changes are either too low or too high, suggesting some 1106 non-linear models. Moreover, the neurovascular system includes different aspects like 1107 excitatory and inhibitory neurons, glial cells, the vasculature components like pericytes 1108 (Populations, 2017). The interaction between inhibitory and excitatory neurons, the glial cell 1109 mediated signaling pathways, and their role in neurovascular coupling have been simplified 1110 in this linear model. A more detailed metabolism model involving blood flow dynamics 1111 (Buxton, 2021) may improve our inferences by comparing it with the model proposed in this 1112 study. Considering such advanced model comparisons, applied within a Bayesian framework, 1113 could be of great interest but was out of the scope of this study. Moreover, we conducted 1114 TMS following the recommendations of the International Federation of Clinical 1115 Neurophysiology (Rossi et al., 2009), which means our data set should not explore extreme 1116 conditions between excitability and hemodynamic response, which are more likely to exhibit 1117 eventual nonlinear relationships.

1118 Another limitation of our study was that the M1 excitability was not assessed at the same 1119 time as the finger-tapping task, but sequentially, hence we did not propose a fusion model to 1120 pool the relationship between cortical excitability and hemodynamic responses at the single1121 trial level. We considered the mean and variance of MEP amplitudes and $\mathrm{HbO} / \mathrm{HbR}$ time 1122 courses within the whole session as the input for the correlation analysis. This might reduce 1123 the resulted correlation values considering additional fluctuations of the baseline excitability 1124 and hemodynamic responses. However, since it has been shown that PAS modulated 1125 excitability changes could last for more than 30 minutes (Stefan, 2000; Lee et al., 2017), we 1126 are confident that our investigation of cortical excitability using MEP after spTMS and 1127 hemodynamic responses elicited by finger tapping was indeed still within this PAS effective 1128 duration window.

1129 As perspectives for this study, it would be of great interest to investigate the relationship 1130 between spTMS evoked $\mathrm{HbO} / \mathrm{HbR}$ and the corresponding MEP amplitude, when occurring 1131 exactly at the same time, therefore, preventing confounds introduced by fluctuations of 1132 excitability and hemodynamic responses along the time. Such an investigation may help us in 1133 understanding the integrity of neurovascular coupling during the transit cortical excitability 1134 change induced by spTMS. Furthermore, the effect of stable cortical excitability changes 1135 (induced by PAS) on this integrity can be explored by comparing the spTMS evoked 1136 hemodynamic responses before and after PAS interventions. Additionally, since fNIRS data 
1137 were recorded during the whole experiments (i.e., also during spTMS and PAS intervention),

1138 our data would allow assessing dynamically the evolution of MEP and hemodynamic

1139 responses during PAS. However, such analysis would require modeling fNIRS response

1140 using advanced deconvolution techniques to handle the overlapping of TMS pulses induced

1141 hemodynamic responses (Machado et al., 2021), and will be considered in our future

1142 investigations.

\section{5. Conclusion}

1144 In this study, we proposed hierarchical Bayesian modeling to investigate the relationship 1145 between motor task-related hemodynamic responses and M1 excitability. When compared 1146 with a sham control condition, a substantial M1 excitability increase was found after PAS25, 1147 and a subtle reduction of M1 excitability was found after PAS10. PAS effects on motor task1148 related hemodynamic responses were observed mainly around the peak of $\mathrm{HbO} / \mathrm{HbR}$ time 1149 courses. We showed a large probability of positive correlations between PAS effects on MEP 1150 amplitudes and hemodynamic responses. Such correlations were also mainly exhibited 1151 around the peak of $\mathrm{HbO} / \mathrm{HbR}$ time courses. Diagnostics of sampling MCMC chains showed 1152 no pathological behavior, ensuring the reliability of our results. Finally, this study also 1153 demonstrated the power of the Bayesian data analysis when dealing with relatively high 1154 variability and small sample size data while providing informative inferences.

\section{Acknowledgments}

1156 This work was supported by the Natural Sciences and Engineering Research Council of 1157 Canada Discovery Grant Program (CG and JML), an operating grant from the Canadian 1158 Institutes for Health Research (CIHR MOP 133619 (CG)), a FRQNT research team grant and 1159 a FRQS-Quebec Bio-Imaging Network (QBIN) Pilot Project grant. fNIRS equipment was 1160 acquired using grants from NSERC Research Tools and Instrumentation Program and the 1161 Canadian Foundation for Innovation (CG). ZC is funded by the Fonds de recherche du 1162 Québec - Sante (FRQS) Doctoral Training Scholarship and the PERFORM Graduate 1163 Scholarship in Preventive Health Research. GP is funded by Strauss Canada Foundation and 1164 a McGill/MNI - Fred Andermann EEG and Epilepsy Fellowship.

\section{Conflict of interest}

1166 The authors declare no potential conflict of interest. 


\section{References}

1169 Aarabi, A., Osharina, V., Wallois, F., 2017. Effect of confounding variables on hemodynamic

1170 response function estimation using averaging and deconvolution analysis: An event-

1171 related NIRS study. Neuroimage 155, 25-49.

1172 https://doi.org/10.1016/j.neuroimage.2017.04.048

1173 Ah Sen, C.B., Fassett, H.J., El-Sayes, J., Turco, C. V, Hameer, M.M., Nelson, A.J., 2017.

$1174 \quad$ Active and resting motor threshold are efficiently obtained with adaptive threshold

1175 hunting. PLoS One 12. https://doi.org/10.1371/journal.pone.0186007

1176 Ahn, W.-Y., Haines, N., Zhang, L., 2017. Revealing Neurocomputational Mechanisms of

1177 Reinforcement Learning and Decision-Making With the hBayesDM Package. Comput.

1178 Psychiatry 1, 24. https://doi.org/10.1162/cpsy_a_00002

1179 Allen, E.A., Pasley, B.N., Duong, T., Freeman, R.D., 2007. Transcranial magnetic

1180 stimulation elicits coupled neural and hemodynamic consequences. Science (80-. ). 317 ,

1181 1918-1921. https://doi.org/10.1126/science.1146426

1182 Arridge, S.R., 1999. Optical tomography in medical imaging. Inverse Probl. 15, R41.

1183 https://doi.org/10.1088/0266-5611/15/2/022

1184 Awiszus, F., Feistner, H., Urbach, D., Bostock, H., 1999. Characterisation of paired-pulse

1185 transcranial magnetic stimulation conditions yielding intracortical inhibition or I-wave

1186 facilitation using a threshold-hunting paradigm. Exp. Brain Res. 129, 317-324.

1187 https://doi.org/10.1007/s002210050901

1188 Bandettini, P.A., Wong, E.C., Hinks, R.S., Tikofsky, R.S., Hyde, J.S., 1992. Time course EPI

1189 of human brain function during task activation. Magn. Reson. Med. 25, 390-397.

1190 https://doi.org/10.1002/mrm.1910250220

1191 Betancourt, M., 2019. The Convergence of Markov Chain Monte Carlo Methods: From the

1192 Metropolis Method to Hamiltonian Monte Carlo. Ann. Phys. 531, 1-6.

$1193 \quad$ https://doi.org/10.1002/andp.201700214

1194 Betancourt, M., 2017. A Conceptual Introduction to Hamiltonian Monte Carlo. eprint

1195 arXiv:1701.02434 60. https://doi.org/https://arxiv.org/abs/1701.02434

1196 Betancourt, M., 2016. Diagnosing Suboptimal Cotangent Disintegrations in Hamiltonian 1197 Monte Carlo. arXiv 1604.00695. 
1198 Betancourt, M., Byrne, S., Livingstone, S., Girolami, M., 2017. The geometric foundations of 1199 Hamiltonian Monte Carlo. Bernoulli 23, 2257-2298. https://doi.org/10.3150/16-BEJ810

1200 Betancourt, M., Girolami, M., 2015. Hamiltonian Monte Carlo for Hierarchical Models. Curr. 1201 Trends Bayesian Methodol. with Appl. 79-101. https://doi.org/10.1201/b18502-5

1202 Bhalerao, S., Kadam, P., 2010. Sample size calculation. Int. J. Ayurveda Res. 1, 55.

1203 https://doi.org/10.4103/0974-7788.59946

1204 Boor, C. de, 2001. A Practical Guide to Splines - Revised Edition, Springer-Verlag, New 1205 York.

1206 Brooks, S.P., Gelman, A., 1998. General methods for monitoring convergence of iterative 1207 simulations. J. Comput. Graph. Stat. 7, 434-455.

$1208 \quad$ https://doi.org/10.1080/10618600.1998.10474787

1209 Buxton, R.B., 2021. The thermodynamics of thinking: Connections between neural activity, 1210 energy metabolism and blood flow: Thermodynamics of thinking. Philos. Trans. R. Soc. 1211 B Biol. Sci. 376. https://doi.org/10.1098/rstb.2019.0624rstb20190624

1212 Cai, Z., Machado, A., Chowdhury, R.A., Spilkin, A., Vincent, T., Aydin, U., Pellegrino, G., 1213 Lina, J.-M., Grova, C., 2021a. Diffuse optical reconstructions of NIRS data using 1214 Maximum Entropy on the Mean. bioRxiv. https://doi.org/10.1101/2021.02.22.432263

1215 Cai, Z., Pellegrino, G., Spilkin, A., Delaire, E., Uji, M., Abdallah, C., Lina, J., Fecteau, S., 1216 Grova, C., 2021b. Hemodynamic Correlates of Fluctuations in Neuronal Excitability $\square$ : 1217 A Simultaneous Paired Associative Stimulation ( PAS ) and functional Near Infra-Red 1218 Spectroscopy ( fNIRS ) Study. bioRxiv. https://doi.org/10.1101/2021.09.29.462418

1219 Cai, Z., Uji, M., Aydin, Ü., Pellegrino, G., Spilkin, A., Delaire, É., Abdallah, C., Lina, J., 1220 Grova, C., 2021c. Evaluation of a personalized functional near infra-red optical tomography workflow using maximum entropy on the mean. Hum. Brain Mapp.

Carson, R.G., Kennedy, N.C., 2013. Modulation of human corticospinal excitability by paired associative stimulation. Front. Hum. Neurosci. 7, 1-28. https://doi.org/10.3389/fnhum.2013.00823 
1229 Chen, G., Padmala, S., Chen, Y., Taylor, P.A., Cox, R.W., Pessoa, L., 2021. To pool or not to pool: Can we ignore cross-trial variability in FMRI? Neuroimage 225, 117496. https://doi.org/10.1016/j.neuroimage.2020.117496

Chen, G., Xiao, Y., Taylor, P.A., Rajendra, J.K., Riggins, T., Geng, F., Redcay, E., Cox, R.W., 2019b. Handling Multiplicity in Neuroimaging Through Bayesian Lenses with Multilevel Modeling. Neuroinformatics 17, 515-545. https://doi.org/10.1007/s12021018-9409-6

Chiang, T.C., Vaithianathan, T., Leung, T., Lavidor, M., Walsh, V., Delpy, D.T., 2007.

Chowdhury, R.A., Lina, J.M., Kobayashi, E., Grova, C., 2013. MEG Source Localization of Spatially Extended Generators of Epileptic Activity: Comparing Entropic and Hierarchical Bayesian Approaches. PLoS One 8, e55969. https://doi.org/10.1371/journal.pone.0055969

Chowdhury, R.A., Merlet, I., Birot, G., Kobayashi, E., Nica, A., Biraben, A., Wendling, F., Lina, J.M., Albera, L., Grova, C., 2016. Complex patterns of spatially extended generators of epileptic activity: Comparison of source localization methods cMEM and 4-ExSo-MUSIC on high resolution EEG and MEG data. Neuroimage 143, 175-195. https://doi.org/10.1016/j.neuroimage.2016.08.044

Corp, D.T., Bereznicki, H.G., Clark, G.M., Youssef, G.J., Fried, P.J., Jannati, A., Davies, C.B., Gomes-Osman, J., Kirkovski, M., Albein-Urios, N., Fitzgerald, P.B., Koch, G., Di Lazzaro, V., Pascual-Leone, A., Enticott, P.G., 2021. Large-scale analysis of interindividual variability in single and paired-pulse TMS data. Clin. Neurophysiol. https://doi.org/10.1016/j.clinph.2021.06.014

Curtin, A., Tong, S., Sun, J., Wang, J., Onaral, B., Ayaz, H., 2019. A systematic review of integrated functional near-infrared spectroscopy (fNIRS) and transcranial magnetic stimulation (TMS) studies. Front. Neurosci. 13. https://doi.org/10.3389/fnins.2019.00084

Dai, N., Kang, H., Jones, G.L., Fiecas, M.B., 2021. A Bayesian latent spatial model for mapping the cortical signature of progression to Alzheimer's disease. Can. J. Stat. 49, 46-62. https://doi.org/10.1002/cjs.11588 
1261 Di Pino, G., Pellegrino, G., Assenza, G., Capone, F., Ferreri, F., Formica, D., Ranieri, F., Tombini, M., Ziemann, U., Rothwell, J.C., Di Lazzaro, V., 2014. Modulation of brain plasticity in stroke: A novel model for neurorehabilitation. Nat. Rev. Neurol. 10, 597608. https://doi.org/10.1038/nrneurol.2014.162

Duane, S., Kennedy, A.D., Pendleton, B.J., Roweth, D., 1987. Hybrid Monte Carlo. Phys. Lett. B 195, 216-222. https://doi.org/10.1016/0370-2693(87)91197-X

Eggebrecht, A.T., White, B.R., Ferradal, S.L., Chen, C., Zhan, Y., Snyder, A.Z., Dehghani,

Eilers, P.H.C., Marx, B.D., 2010. Splines, knots, and penalties. Wiley Interdiscip. Rev. Comput. Stat. 2, 637-653. https://doi.org/10.1002/wics.125

Fang, Q., Boas, D.A., 2009. Monte Carlo Simulation of Photon Migration in 3D Turbid Media Accelerated by Graphics Processing Units. Opt. Express 17, 20178. https://doi.org/10.1364/oe.17.020178

Fischl, B., Salat, D.H., Busa, E., Albert, M., Dieterich, M., Haselgrove, C., Van Der Kouwe, A.M., 2002. Whole brain segmentation: Automated labeling of neuroanatomical structures in the human brain. Neuron 33, 341-355. https://doi.org/10.1016/S0896-

Fonov, V., Evans, A.C., Botteron, K., Almli, C.R., McKinstry, R.C., Collins, D.L., 2011. 6273(02)00569-X Unbiased average age-appropriate atlases for pediatric studies. Neuroimage 54, 313 327. https://doi.org/10.1016/j.neuroimage.2010.07.033

Gabry, J., Mahr, T., 2020. bayesplot: Plotting for Bayesian Models. 
1292

1293

1294

1295

1296

1297

1298

1299

1300

1301

1302

1303

1304

1305

1306

1307

1308

1309

1310

1311

1312

1313

1314

1315

1316

1317

1318

1319

1320

1321

densities. J. Am. Stat. Assoc. 85, 398-409.

https://doi.org/10.1080/01621459.1990.10476213

Gelman, A., Carlin, J., 2014. Beyond Power Calculations: Assessing Type S (Sign) and Type M (Magnitude) Errors. Perspect. Psychol. Sci. 9, 641-651.

https://doi.org/10.1177/1745691614551642

Gelman, A., Carlin, J.B., Stern, H.S., Dunson, D.B., Vehtari, A., Rubin, D.B., 2013. Bayesian Data Analysis Third edition, 3rd ed. CRC press.

Gelman, A., Hill, J., Vehtari, A., 2020a. Regression and other stories. Cambridge University Press.

Gelman, A., Jakulin, A., Pittau, M.G., Su, Y.S., 2008. A weakly informative default prior distribution for logistic and other regression models. Ann. Appl. Stat. 2, 1360-1383. https://doi.org/10.1214/08-AOAS191

Gelman, A., Rubin, D.B., 1992. Inference from iterative simulation using multiple sequences. Stat. Sci. 7, 457-472. https://doi.org/10.1214/ss/1177011136

Gelman, A., Simpson, D., Betancourt, M., 2017. The prior can often only be understood in the context of the likelihood. Entropy 19, 1-13. https://doi.org/10.3390/e19100555

Gelman, A., Vehtari, A., Simpson, D., Margossian, C.C., Carpenter, B., Yao, Y., Kennedy, L., Gabry, J., Bürkner, P.C., Modrák, M., 2020b. Bayesian workflow. arXiv.

Geman, S., Geman, D., 1984. Stochastic Relaxation, Gibbs Distributions, and the Bayesian Restoration of Images. IEEE Trans. Pattern Anal. Mach. Intell. PAMI-6, 721-741. https://doi.org/10.1109/TPAMI.1984.4767596

Glover, G.H., 2011. Overview of functional magnetic resonance imaging. Neurosurg. Clin. N. Am. https://doi.org/10.1016/j.nec.2010.11.001

Grova, C., Aiguabella, M., Zelmann, R., Lina, J.M., Hall, J.A., Kobayashi, E., 2016. Intracranial EEG potentials estimated from MEG sources: A new approach to correlate MEG and iEEG data in epilepsy. Hum. Brain Mapp. 37, 1661-1683. https://doi.org/10.1002/hbm.23127

Grova, C., Makni, S., Flandin, G., Ciuciu, P., Gotman, J., Poline, J.B., 2006. Anatomically informed interpolation of fMRI data on the cortical surface. Neuroimage 31, 1475-1486. https://doi.org/10.1016/j.neuroimage.2006.02.049 
1322 Hashemi, M., Vattikonda, A.N., Sip, V., Guye, M., Bartolomei, F., Woodman, M.M., Jirsa, V.K., 2020. The Bayesian Virtual Epileptic Patient: A probabilistic framework designed to infer the spatial map of epileptogenicity in a personalized large-scale brain model of epilepsy spread. Neuroimage 217, 116839. https://doi.org/10.1016/j.neuroimage.2020.116839

Hastie, T.J., 2017. Generalized additive models, in: Statistical Models in S. https://doi.org/10.1201/9780203738535

Hattemer, K., Knake, S., Reis, J., Rochon, J., Oertel, W.H., Rosenow, F., Hamer, H.M., 2007. Excitability of the motor cortex during ovulatory and anovulatory cycles: A transcranial magnetic stimulation study. Clin. Endocrinol. (Oxf). 66, 387-393. https://doi.org/10.1111/j.1365-2265.2007.02744.x

Hedrich, T., Pellegrino, G., Kobayashi, E., Lina, J.M., Grova, C., 2017. Comparison of the spatial resolution of source imaging techniques in high-density EEG and MEG. Neuroimage 157, 531-544. https://doi.org/10.1016/j.neuroimage.2017.06.022

Heers, M., Chowdhury, R.A., Hedrich, T., Dubeau, F., Hall, J.A., Lina, J.M., Grova, C., Kobayashi, E., 2016. Localization Accuracy of Distributed Inverse Solutions for Electric and Magnetic Source Imaging of Interictal Epileptic Discharges in Patients with Focal Epilepsy. Brain Topogr. 29, 162-181. https://doi.org/10.1007/s10548-014-0423-1 Differences in the hemodynamic response to event-related motor and visual paradigms

Jöbsis, F.F., 1977. Noninvasive, infrared monitoring of cerebral and myocardial oxygen sufficiency and circulatory parameters. Science (80-. ). 198, 1264-1266. https://doi.org/10.1126/science.929199

Kay, M., 2020. tidybayes: Tidy Data and Geoms for Bayesian Models. R package version 2.3.0. https//mjskay.github.io/tidybayes/. DOI 10.5281/zenodo.1308151. https://doi.org/10.5281/zenodo.1308151 induced changes in MEP amplitude and BOLD signal. Brain Stimul. 6, 330-339. 
1353 Kwong, K.K., Belliveau, J.W., Chesler, D.A., Goldberg, I.E., Weisskoff, R.M., Poncelet, B.P., Kennedy, D.N., Hoppel, B.E., Cohen, M.S., Turner, R., Cheng -, H.M., Brady, T.J., Rosen, B.R., 1992. Dynamic magnetic resonance imaging of human brain activity during primary sensory stimulation. Proc. Natl. Acad. Sci. U. S. A. 89, 5675-5679. https://doi.org/10.1073/pnas.89.12.5675

Lee, J.C., Croarkin, P.E., Ameis, S.H., Sun, Y., Blumberger, D.M., Rajji, T.K., Daskalakis, Cortex Plasticity in Healthy Adolescents. Front. Psychiatry 8, 1-8. https://doi.org/10.3389/fpsyt.2017.00095

Levy, W.B., Steward, O., 1983. Temporal contiguity requirements for long-term associative potentiation/depression in the hippocampus. Neuroscience 8, 791-797. https://doi.org/10.1016/0306-4522(83)90010-6

Lewandowski, D., Kurowicka, D., Joe, H., 2009. Generating random correlation matrices based on vines and extended onion method. J. Multivar. Anal. 100, 1989-2001. https://doi.org/10.1016/j.jmva.2009.04.008

López-Alonso, V., Cheeran, B., Río-Rodríguez, D., Fernández-Del-Olmo, M., 2014. Interindividual variability in response to non-invasive brain stimulation paradigms. Brain Stimul. 7, 372-380. https://doi.org/10.1016/j.brs.2014.02.004

Machado, A., Cai, Z., Pellegrino, G., Marcotte, O., Vincent, T., Lina, J.-M., Kobayashi, E., Grova, C., 2018. Optimal positioning of optodes on the scalp for personalized functional

Machado, A., Cai, Z., Vincent, T., Pellegrino, G., Lina, J.M., Kobayashi, E., Grova, C., 2021. Deconvolution of hemodynamic responses along the cortical surface using personalized functional near infrared spectroscopy. Sci. Rep. 1-19. https://doi.org/10.1038/s41598021-85386-0 montage on electroencephalography/functional near-infrared spectroscopy caps dedicated to study epileptic discharges. J. Biomed. Opt. 19, 026010.

1383 Mariorenzi, R., Zarola, F., Caramia, M.D., Paradiso, C., Rossini, P.M., 1991. Non-invasive 
1384

1385

1386

1387

1388

1389

1390

1391

1392

1393

1394

1395

1396

1397

1398

1399

1400

1401

1402

1403

1404

1405

1406

1407

1408

1409

1410

1411

1412

1413

1414

evaluation of central motor tract excitability changes following peripheral nerve stimulation in healthy humans. Electroencephalogr. Clin. Neurophysiol. Evoked Potentials 81, 90-101. https://doi.org/10.1016/0168-5597(91)90002-F

McElreath, R., 2020. Statistical Rethinking, 2nd ed, CRC Press. https://doi.org/10.1201/9781315372495

Näsi, T., Mäki, H., Kotilahti, K., Nissilä, I., Haapalahti, P., Ilmoniemi, R.J., 2011. Magneticstimulation-related physiological artifacts in hemodynamic near-infrared spectroscopy signals. PLoS One 6. https://doi.org/10.1371/journal.pone.0024002

Navarro De Lara, L.I., Windischberger, C., Kuehne, A., Woletz, M., Sieg, J., Bestmann, S., Weiskopf, N., Strasser, B., Moser, E., Laistler, E., 2015. A novel coil array for combined TMS/fMRI experiments at 3 T. Magn. Reson. Med. 74, 1492-1501. https://doi.org/10.1002/mrm.25535

Neal, R.M., 2010. MCMC Using Hamiltonian Dynamics. Handb. Markov Chain Monte Carlo $54,113-162$.

Neal, R.M., 2003. Slice sampling. Ann. Stat. 31, 705-767. https://doi.org/10.2307/3448415

Neal, R.M., 1998. Regression and Classification Using Gaussian Process Priors. Bayesian Stat. 6, 475-501.

Novi, S.L., Forero, E.J., Rubianes Silva, J.A.I., de Souza, N.G.S.R., Martins, G.G., Quiroga, A., Wu, S.T., Mesquita, R.C., 2020. Integration of Spatial Information Increases Reproducibility in Functional Near-Infrared Spectroscopy. Front. Neurosci. 14, 1-12. https://doi.org/10.3389/fnins.2020.00746

Papaspiliopoulos, O., Roberts, G.O., Sköld, M., 2007. A general framework for the parametrization of hierarchical models. Stat. Sci. https://doi.org/10.1214/088342307000000014

Pellegrino, G., Hedrich, T., Porras-Bettancourt, M., Lina, J.M., Aydin, Ü., Hall, J., Grova, C., Kobayashi, E., 2020. Accuracy and spatial properties of distributed magnetic source imaging techniques in the investigation of focal epilepsy patients. Hum. Brain Mapp. 41, 3019-3033. https://doi.org/10.1002/hbm.24994

Pellegrino, G., Machado, A., von Ellenrieder, N., Watanabe, S., Hall, J.A., Lina, J.M., Kobayashi, E., Grova, C., 2016. Hemodynamic response to interictal epileptiform discharges addressed by personalized EEG-fNIRS recordings. Front. Neurosci. 10, 102. 
https://doi.org/10.3389/fnins.2016.00102

1416 Penny, W.D., Friston, K.J., Ashburner, J.T., Kiebel, S.J., Nichols, T.E., 2011. Statistical parametric mapping: the analysis of functional brain images, Elsevier.

Piper, S.K., Krueger, A., Koch, S.P., Mehnert, J., Habermehl, C., Steinbrink, J., Obrig, H., Schmitz, C.H., 2014. A wearable multi-channel fNIRS system for brain imaging in freely moving subjects. Neuroimage 85, 64-71. https://doi.org/10.1016/j.neuroimage.2013.06.062

Populations, T. variation and factors influencing vertical migration behavior in D., 2017. The neurovascular unit coming of age: a journey through neurovascular coupling in health and disease Costantino 96(1): 17-42. doi:10.1016/j.neuron.2017.07.030. The neurovascular unit coming of age: a journe. Physiol. Behav. 176, 139-148. https://doi.org/10.1016/j.neuron.2017.07.030.The

R Core Team, 2020. R: A Language and Environment for Statistical Computing.

Raffin, E., Pellegrino, G., Di Lazzaro, V., Thielscher, A., Siebner, H.R., 2015. Bringing transcranial mapping into shape: Sulcus-aligned mapping captures motor somatotopy in human primary motor hand area. Neuroimage 120, 164-175. https://doi.org/10.1016/j.neuroimage.2015.07.024

Ridding, M.C., Rothwell, J.C., 2007. Is there a future for therapeutic use of transcranial magnetic stimulation? Nat. Rev. Neurosci. https://doi.org/10.1038/nrn2169

Roberts, G.O., Rosenthal, J.S., 2004. General state space Markov chains and MCMC algorithms. Probab. Surv. 1, 20-71. https://doi.org/10.1214/154957804100000024

Rossi, S., Hallett, M., Rossini, P.M., Pascual-Leone, A., Avanzini, G., Bestmann, S., Berardelli, A., Brewer, C., Canli, T., Cantello, R., Chen, R., Classen, J., Demitrack, M., Di Lazzaro, V., Epstein, C.M., George, M.S., Fregni, F., Ilmoniemi, R., Jalinous, R., Karp, B., Lefaucheur, J.P., Lisanby, S., Meunier, S., Miniussi, C., Miranda, P., Padberg, F., Paulus, W., Peterchev, A., Porteri, C., Provost, M., Quartarone, A., Rotenberg, A., Rothwell, J., Ruohonen, J., Siebner, H., Thut, G., Valls-Solè, J., Walsh, V., Ugawa, Y., Zangen, A., Ziemann, U., 2009. Safety, ethical considerations, and application guidelines for the use of transcranial magnetic stimulation in clinical practice and research. Clin. Neurophysiol. https://doi.org/10.1016/j.clinph.2009.08.016 
1446

Ferreri, F., Fitzgerald, P.B., George, M.S., Hallett, M., Lefaucheur, J.P., Langguth, B., Matsumoto, H., Miniussi, C., Nitsche, M.A., Pascual-Leone, A., Paulus, W., Rossi, S., Rothwell, J.C., Siebner, H.R., Ugawa, Y., Walsh, V., Ziemann, U., 2015. Non-invasive electrical and magnetic stimulation of the brain, spinal cord, roots and peripheral nerves: Basic principles and procedures for routine clinical and research application: An updated report from an I.F.C.N. Committee. Clin. Neurophysiol. 126, 1071-1107. https://doi.org/10.1016/j.clinph.2015.02.001

Rubin, D.B., 2004. Multiple imputation for nonresponse in surveys. John Wiley \& Sons.

Schmitz, C.H., Klemer, D.P., Hardin, R., Katz, M.S., Pei, Y., Graber, H.L., Levin, M.B., Levina, R.D., Franco, N.A., Solomon, W.B., Barbour, R.L., 2005. Design and implementation of dynamic near-infrared optical tomographic imaging instrumentation for simultaneous dual-breast measurements. Appl. Opt. 44, 2140. https://doi.org/10.1364/AO.44.002140

Schneider, P., Piper, S., Schmitz, C.H., Schreiter, N.F., Volkwein, N., Lüdemann, L., Malzahn, U., Poellinger, A., 2011. Fast 3D near-infrared breast imaging using indocyanine green for detection and characterization of breast lesions. RoFo Fortschritte auf dem Gebiet der Rontgenstrahlen und der Bildgeb. Verfahren 183, 956-963. https://doi.org/10.1055/s-0031-1281726

Scholkmann, F., Kleiser, S., Metz, A.J., Zimmermann, R., Mata Pavia, J., Wolf, U., Wolf, M., 2014. A review on continuous wave functional near-infrared spectroscopy and imaging instrumentation and methodology. Neuroimage. https://doi.org/10.1016/j.neuroimage.2013.05.004

Seewoo, B.J., Feindel, K.W., Etherington, S.J., Rodger, J., 2019. Frequency-specific effects of low-intensity rTMS can persist for up to 2 weeks post-stimulation: A longitudinal rsfMRI/MRS study in rats. Brain Stimul. 12, 1526-1536. https://doi.org/10.1016/j.brs.2019.06.028

Siebner, H.R., Bergmann, T.O., Bestmann, S., Massimini, M., Johansen-Berg, H., Mochizuki, H., Bohning, D.E., Boorman, E.D., Groppa, S., Miniussi, C., Pascual-Leone, A., Huber, R., Taylor, P.C.J., Ilmoniemi, R.J., De Gennaro, L., Strafella, A.P., Kähkönen, S., Klöppel, S., Frisoni, G.B., George, M.S., Hallett, M., Brandt, S.A., Rushworth, M.F., Ziemann, U., Rothwell, J.C., Ward, N., Cohen, L.G., Baudewig, J., Paus, T., Ugawa, Y., Rossini, P.M., 2009. Consensus paper: Combining transcranial stimulation with 
neuroimaging. Brain Stimul. 2, 58-80. https://doi.org/10.1016/j.brs.2008.11.002

1479 Sotero, R.C., Trujillo-Barreto, N.J., 2007. Modelling the role of excitatory and inhibitory

1480 neuronal activity in the generation of the BOLD signal. Neuroimage 35, 149-165.

$1481 \quad$ https://doi.org/10.1016/j.neuroimage.2006.10.027

1482 Stan Development Team, 2020a. Stan Modeling Language Users Guide and Reference

1483 Manual, 2.25.0.

1484 Stan Development Team, 2020b. RStan: the R interface to Stan, 2.21.2.

1485 Stefan, K., 2000. Induction of plasticity in the human motor cortex by paired associative 1486 stimulation. Brain 123, 572-584. https://doi.org/10.1093/brain/123.3.572

1487 Steinbrink, J., Villringer, A., Kempf, F., Haux, D., Boden, S., Obrig, H., 2006. Illuminating 1488 the BOLD signal: combined fMRI-fNIRS studies. Magn. Reson. Imaging 24, 495-505. 1489 https://doi.org/10.1016/j.mri.2005.12.034

1490 Suppa, A., Quartarone, A., Siebner, H., Chen, R., Di Lazzaro, V., Del Giudice, P., Paulus, 1491 W., Rothwell, J.C., Ziemann, U., Classen, J., 2017. The associative brain at work: 1492 Evidence from paired associative stimulation studies in humans. Clin. Neurophysiol. $1493 \quad 128,2140-2164$. https://doi.org/10.1016/j.clinph.2017.08.003

1494 Tadel, F., Baillet, S., Mosher, J.C., Pantazis, D., Leahy, R.M., 2011. Brainstorm: A user1495 friendly application for MEG/EEG analysis. Comput. Intell. Neurosci. 2011, 879716. 1496 https://doi.org/10.1155/2011/879716

1497 Thomson, R.H., Cleve, T.J., Bailey, N.W., Rogasch, N.C., Maller, J.J., Daskalakis, Z.J., 1498 Fitzgerald, P.B., 2013. Blood oxygenation changes modulated by coil orientation during 1499 prefrontal transcranial magnetic stimulation. Brain Stimul. 6, 576-581.

$1500 \quad$ https://doi.org/10.1016/j.brs.2012.12.001

1501 Tik, M., Hoffmann, A., Sladky, R., Tomova, L., Hummer, A., Navarro de Lara, L., 1502 Bukowski, H., Pripfl, J., Biswal, B., Lamm, C., Windischberger, C., 2017. Towards understanding rTMS mechanism of action: Stimulation of the DLPFC causes networkspecific increase in functional connectivity. Neuroimage 162, 289-296.

1506 Tsang, P., Bailey, A.Z., Nelson, A.J., 2015. Rapid-rate paired associative stimulation over the 1507 primary somatosensory cortex. PLoS One 10, e0120731.

1508 https://doi.org/10.1371/journal.pone.0120731 
1509 Vehtari, A., Gelman, A., Simpson, D., Carpenter, B., Bürkner, P.C., 2020. Rank-

1510 normalization, folding, and localization: An improved $\mathrm{R}$ for assessing convergence of

1511 MCMC. Bayesian Anal. https://doi.org/10.1214/20-ba1221

1512 Ventrucci, M., Rue, H., 2016. Penalized complexity priors for degrees of freedom in

1513 Bayesian P-splines. Stat. Modelling 16, 429-453.

1514 https://doi.org/10.1177/1471082X16659154

1515 Wang, W.T., Xu, B., Butman, J.A., 2017. Improved SNR for combined TMS-fMRI: A

1516 support device for commercially available body array coil. J. Neurosci. Methods 289, 1-

1517 7. https://doi.org/10.1016/j.jneumeth.2017.06.020

1518 Wickham, H., Averick, M., Bryan, J., Chang, W., McGowan, L.D., François, R., Grolemund, 1519 G., Hayes, A., Henry, L., Hester, J., Kuhn, M., Pedersen, T.L., Miller, E., Bache, S.M., Müller, K., Ooms, J., Robinson, D., Seidel, D.P., Spinu, V., Takahashi, K., Vaughan, D., Wilke, C., Woo, K., Yutani, H., 2019. Welcome to the tidyverse. J. Open Source Softw. 4, 1686. https://doi.org/10.21105/joss.01686

Witt, S.T., Laird, A.R., Meyerand, M.E., 2008. Functional neuroimaging correlates of fingertapping task variations: An ALE meta-analysis. Neuroimage 42, 343-356. https://doi.org/10.1016/j.neuroimage.2008.04.025

Wolters, A., Schmidt, A., Schramm, A., Zeller, D., Naumann, M., Kunesch, E., Benecke, R., Reiners, K., Classen, J., 2005. Timing-dependent plasticity in human primary somatosensory cortex. J. Physiol. 565, 1039-1052. magnetic stimulation of the parietal cortex facilitates spatial working memory: Nearinfrared spectroscopy study. Cereb. Cortex 20, 1037-1045. https://doi.org/10.1093/cercor/bhp163 Monte Carlo photon transport simulations for heterogeneous computing platforms. J. Biomed. Opt. 23, 1. https://doi.org/10.1117/1.jbo.23.1.010504 
1540 Yücel, M.A., von Lühmann, A., Scholkmann, F., Gervain, J., Dan, I., Ayaz, H., Boas, D., 1541 Cooper, R.J., Culver, J., Elwell, C.E., Eggebrecht, A., Franceschini, M.A., Grova, C.,

1542 Homae, F., Lesage, F., Obrig, H., Tachtsidis, I., Tak, S., Tong, Y., Torricelli, A.,

1543 Wabnitz, H., Wolf, M., 2020. Best Practices for fNIRS publications. Neurophotonics (in 1544 revisi.

1545 Zeff, B.W., White, B.R., Dehghani, H., Schlaggar, B.L., Culver, J.P., 2007. Retinotopic

1546 mapping of adult human visual cortex with high-density diffuse optical tomography.

1547 Proc. Natl. Acad. Sci. 104, 12169-12174. https://doi.org/10.1073/pnas.0611266104 


\section{Appendices}

\section{Appendix 1 fNIRS data processing}

1552 Raw fNIRS data were first preprocessed following standard recommendations (Yücel et al.,

1553 2020): a) bad channel rejections of channels exhibiting either a negative raw amplitude 1554 during the whole time course and a coefficient of variation (CV) larger than 8\% (Schmitz et 1555 al., 2005; Schneider et al., 2011; Eggebrecht et al., 2012; Piper et al., 2014): b) linear 1556 regression of superficial physiological fluctuations using the average of all proximity 1557 channels (Zeff et al., 2007); c) band-pass filtering (i.e., $0.01 \mathrm{~Hz}$ to $0.1 \mathrm{~Hz}$ ) using a $3 \mathrm{rd}$ order 1558 Butterworth filter (zero-phase); d) conversion in optical density changes (i.e., $\Delta \mathrm{OD}$ ) using 1559 logarithm conversion; e) $\triangle \mathrm{OD}$ epochs extraction within a time window ranging from -10s to 156030 s around task onsets. Instead of the conventional process averaging extracted $\Delta \mathrm{OD}$ epochs, 1561 we then conducted a resampling process to estimate not one but a set of 'possible' averaged $1562 \Delta$ ODs (Cai et al., 2021b). Our rationale was to propose an evaluation preserving the intrinsic 1563 variance of averaged $\triangle \mathrm{OD}$ related to the underlying physiological fluctuations and eventual 1564 measurement errors such as motion artifacts. To do so, we first averaged 16 out of 20 1565 preprocessed $\triangle \mathrm{OD}$ epochs for all possible unique combinations (i.e., $C_{20}^{16}=4845$ 1566 possibilities). Then, the averaged signal to noise ratio (SNR) of the resulting averaged $\Delta$ ODs, 1567 for each wavelength, was estimated as the peak amplitude over the averaged standard 1568 deviation of baseline (within -10s to 0s) among all channels. Lastly, we selected 101 of these 1569 resampled averaged $\triangle \mathrm{ODs}$, distributed around the median SNR (50 averaged below and 50 1570 averaged above the median SNR), to obtain a distribution of 'possible' responses evoked by 1571 one finger-tapping run. The selection of 16 blocks out of 20 trials and 101 resampled 1572 averaged $\triangle$ ODs maintained a good coverage of the data distribution. This number was 1573 empirically defined according to the observation that usually there were less than four blocks 1574 contaminated with artifacts in one finger-tapping run. Selecting sub-averaged trials around 1575 the median SNR ensured a good representation of fNIRS responses while discarding artifacts 1576 in the meantime. Indeed, in artifacts contaminated data, large motion artifacts would result in 1577 high SNR of corresponding sub-averaged trials.

1578 We then applied 3D fNIRS reconstruction workflow using personalized optimal montage and 1579 maximum entropy on the mean (MEM), as further described and validated in our earlier work 1580 (Cai et al., 2021c), to the 101 sub-averaged $\Delta$ ODs. Therefore, 'all possible' $\mathrm{HbO} / \mathrm{HbR}$ 1581 responses for each finger-tapping run were reconstructed as spatiotemporal maps along the 
1582 cortical surface. To do so, the subject-specific fNIRS forward model was first estimated

1583 according to the following steps: a) 5 tissues head segmentation (e.g., scalp, skull,

1584 Cerebrospinal fluid, grey matter and white matter) calculated using FreeSurfer6.0 (Fischl et 1585 al., 2002) (https://surfer.nmr.mgh.harvard.edu) and SPM12 (Penny et al., 2011) 1586 (https://www.fil.ion.ucl.ac.uk/spm/software/spm12/); b) light fluences at each optode 1587 location, and for each wavelength (i.e., $685 \mathrm{~nm}$ and $830 \mathrm{~nm}$ ), were calculated by simulating $158810^{8}$ photons, using MCXLAB toolbox - a Monte Carlo photon simulator for modeling light 1589 transport in 3D turbid media, developed by Fang and Boas, 2009 and Yu et al., 2018; c) 1590 sensitivity of each voxel was computed using the adjoint formulation and was normalized by 1591 Rytov approximation (Arridge, 1999); d) surface space sensitivity was finally obtained by 1592 projecting volumetric sensitivity map to subject's cortical surface (i.e., mid-surface, a middle 1593 layer of the gray matter, between pia mater and gray-white matter interface, 25,000 vertices) 1594 using the Voronoi based method proposed by Grova et al., 2006. Finally, each of 101 1595 averaged $\triangle \mathrm{OD}$ epoch was down-sampled to $2 \mathrm{~Hz}$ and MEM method proposed previously by 1596 our group for fNIRS reconstruction (Cai et al., 2021a, 2021c) was applied to estimate the $1597 \mathrm{HbO} / \mathrm{HbR}$ spatiotemporal maps (0s to 30s) along the subject-specific cortical surface. 


\section{Supplementary material}
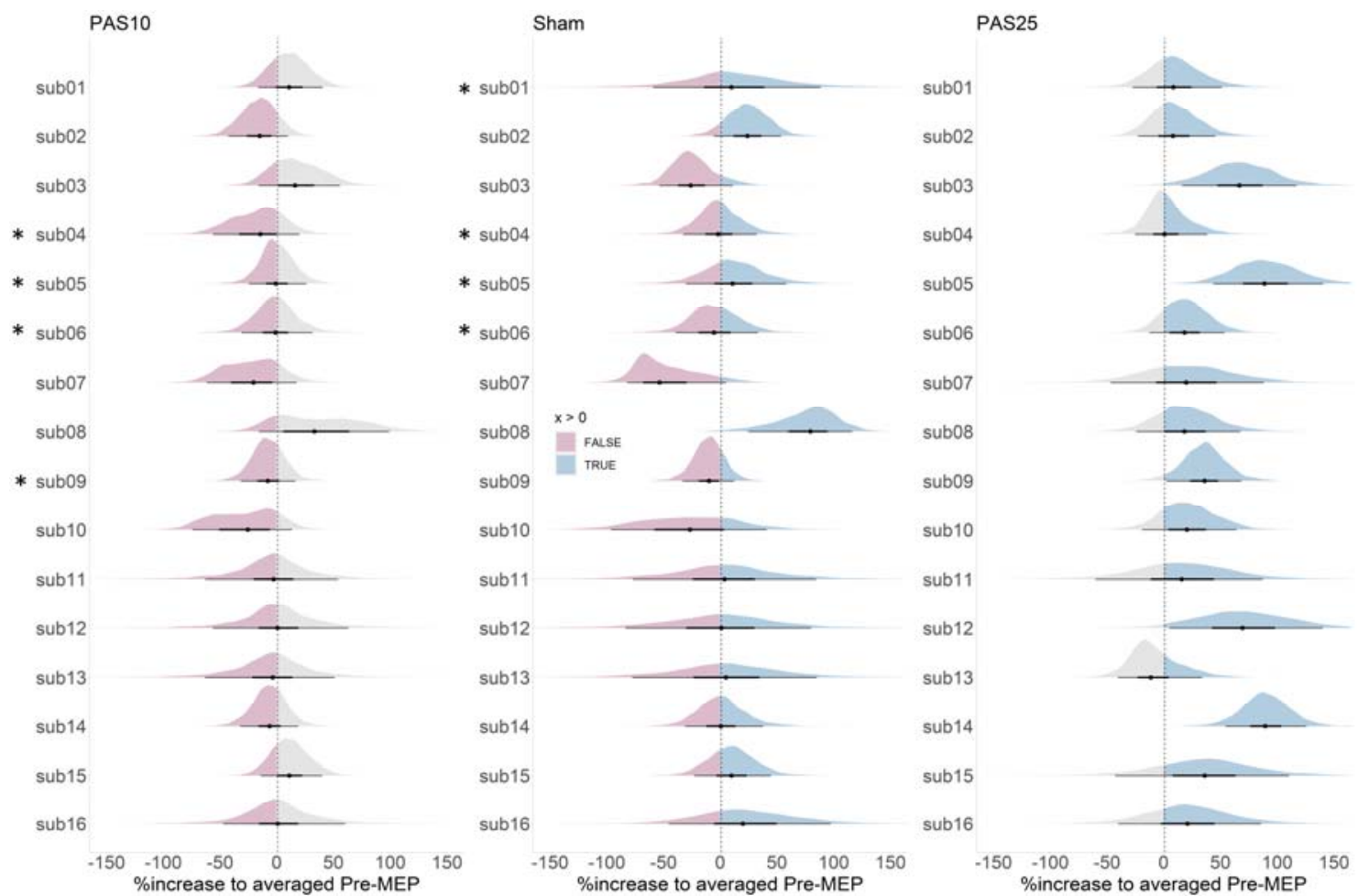

1601 Fig.S1. PAS effects on M1 excitability at the individual level. Each column presents the individual

1602 level posterior distribution of the intervention effect from PAS10, sham and PAS25, respectively.

1603 Posterior predictive simulations of post-PAS MEP amplitudes were conducted by assuming the same

1604 pre-PAS MEP amplitude for all subjects, i.e., the averaged pre-PAS MEP amplitude obtained for all

1605 subjects over all 40 sessions. The blue area represents the probability of obtaining a relative increase

1606 (in\%) for the post-PAS MEP amplitude when compared to the pre-PAS MEP amplitude, whereas the

1607 pink area represents the probability of obtaining a relative decrease (in \%). The black dot represents

1608 the median of each posterior distribution, and the surrounding bars show the corresponding 50\% and

$160990 \%$ credibility intervals. Overall, large between-subject variability can be observed for both

1610 interventions. Note that missing sessions were also included using posterior predictive simulations

1611 within the model, based on prior distributions and partial pooled information from other sessions. *

1612 indicate the missing runs that were imputed by the model. 